OPEN ACCESS

Edited by:

Francesca Gay,

University Hospital of the City of Health and Science of Turin, Italy

Reviewed by:

Frederique Vegran

INSERM U1231 Lipides, Nutrition,

Cancer (LNC), France

Kawaljit Kaur,

University of California, Los Angeles,

United States

*Correspondence:

Paola Neri

epneri@ucalgary.ca

Specialty section

This article was submitted to

Cancer Immunity and Immunotherapy,

a section of the journal

Frontiers in Oncology

Received: 13 January 2020 Accepted: 06 April 2020

Published: 05 May 2020

Citation:

Leblay N, Maity R, Hasan F and Neri P (2020) Deregulation of Adaptive T Cell

Immunity in Multiple Myeloma: Insights

Into Mechanisms and Therapeutic

Opportunities. Front. Oncol. 10:636.

doi: 10.3389/fonc.2020.00636

\section{Deregulation of Adaptive T Cell Immunity in Multiple Myeloma: Insights Into Mechanisms and Therapeutic Opportunities}

\author{
Noémie Leblay, Ranjan Maity, Fajer Hasan and Paola Neri* \\ Arnie Charbonneau Cancer Institute, University of Calgary, Calgary, AB, Canada
}

Immunotherapy has recently emerged as a promising treatment option for multiple myeloma (MM) patients. Profound immune dysfunction and evasion of immune surveillance are known to characterize MM evolution and disease progression. Along with genomic changes observed in malignant plasma cells, the bone marrow (BM) milieu creates a protective environment sustained by the complex interaction of BM stromal cells (BMSCs) and malignant cells that using bidirectional connections and cytokines released stimulate disease progression, drug resistance and enable immune escape. Local immune suppression and T-cell exhaustion are important mediating factors of clinical outcomes and responses to immune-based approaches. Thus, further characterization of the defects present in the immune system of MM patients is essential to develop novel therapies and to repurpose the existing ones. This review seeks to provide insights into the mechanisms that promote tumor escape, cause inadequate T-cell stimulation and impaired cytotoxicity in MM. Furthermore, it highlights current immunotherapies being used to restore adaptive T-cell immune responses in MM and describes strategies created to escape these multiple immune evasion mechanisms.

Keywords: multiple myeloma, immunotherapy, bone marrow microenviroment, monoclonal antibodies, T-cell therapies

\section{INTRODUCTION}

Multiple myeloma (MM), although a rare disease, is the second most common hematologic malignancy (1) with over 130,000 new cases occurring every year globally (2). It is a cancer of plasma cells, resulting from abnormal growth of malignant plasma cells in the bone marrow (BM) (3). MM is associated with impaired immunity and immune dysregulation. As such the Bcell dysfunction is characterized by immunoparesis, hypo-gammaglobulinemia of the uninvolved immunoglobulins and increased susceptibility to infections (4). Deficiencies in T-cell function and tissue distribution have been also reported in MM (5). A well-recognized feature of MM is also the bidirectional relationship between the tumor plasma cells and the BM milieu, which provides a protective niche promoting MM tumor growth and loss of immune surveillance (6). Although the advent of novel therapies has improved the outcomes of MM patients $(7,8)$, the majority of them will relapse and became refractory to current therapy. Therefore, innovative therapeutic strategies, such as immunotherapy, have been established to improve the survival of these patients $(9,10)$. In the last ten years, a deeper insight into MM biology and its immune defects alongside with 
the development of numerous immune-based therapies have allowed immunotherapy to become a promising new treatment option for MM patients $(9,10)$.

As such three major anti-MM immunotherapeutic approaches have been developed: (i) agents that remove the breaks of the immune system, such as immunomodulatory agents (IMiDs) and immune checkpoint inhibitors, (ii) agents that target highly selective antigens on the MM cells in the form of monoclonal

\footnotetext{
Abbreviations: ADCC, Antibody driven cellular cytotoxicity; ADCP, Antibody mediated cellular phagocytosis; ADCs, Antibody-drug conjugates; AEs, Adverse events; ALL, Acute lymphoblastic leukemia; APCs, Antigen presenting cells; APRIL, A proliferation inducing ligand; ARG1, Arginase; ASCT, Autologous stem cell transplant; ASH, American Society of Hematology; BAFF, B-Cell Activating Factor; BCMA, B-cell maturation antigen; BiTEs, Bispecific T-cell engagers; BM, Bone marrow; BMSCs, Bone marrow stromal cells; CAR, Chimeric antigen receptor; CDC, Complement dependent cytotoxicity; CK1 $\alpha$, Casein kinase $1 \alpha$; COX2, Cyclooxygenase-2; CR, Complete response; CRBN, Cereblon; CRS, Cytokine release syndrome; CTL, Cytotoxic T lymphocyte; CTLA-4, Cytotoxic T-lymphocyte-associated antigen 4; DC, Dendritic cell; EMA, European Medicines Agency; FDA, Food and Drug Administration; FOXP3, Forkhead box P3; GPCR, G protein-coupled receptor; GPRC5D, Class C group 5 member D; His6-tag, Hexa-histidine; IDO, Indoleamine 2,3-dioxygenase; IFN $\gamma$, Interferon gamma; Ig, Immunoglobulin; IKZF1, IKAROS Family Zinc Finger 1; IKZF3, AIOLOS; IL, Interleukin; IMiDs, Immunomodulatory agents; iNOS, Inducible nitric oxygen synthase; IRF4, Interferon regulatory factor 4; ITIM, Immunoreceptor tyrosine based-inhibition motif; JNK, c-Jun N-terminal kinase; LAG3, Lymphocyteactivation gene 3; mAbs, Monoclonal antibodies; MAPK8, Mitogen-Activated Protein Kinase 8; MDSCs, Myeloid-derived suppressor cells; MGUS, Monoclonal Gammopathy of Undetermined Significance; MHC, Major histocompatibility complex; MM, Multiple myeloma; mOS, Median overall survival; mPFS, Median progression-free survival; MRD, Minimal Residual Disease; MTD, Maximum tolerated dose; NCI, National Cancer Institute; nCR, Near complete response; NK, Natural killer; NDMM, Newly diagnosed multiple myeloma; NOS, Nitric oxide synthase; ORRs, Overall response rates; OS, Overall survival; PD-L1, Programmed death-ligand 1; PD1, Programmed death 1; pDCs, Plasmacytoid dendritic cells; PFS, Progression-free survival; PI3K, Phosphoinositide 3 kinase; PIs, Proteasome inhibitors; PR, Partial response; RANKL, Receptor activator of nuclear factor kappa-B ligand; ROS, Reactive species of oxygen; RRMM, Relapsed refractory multiple myeloma; scFv, Single-chain variable fragment; $\mathrm{sCR}$, Stringent complete remission; SCT, Stem cell transplantation; SLAMF7, Signaling lymphocytic activation molecule F7; SMM, smoldering multiple myeloma; sMICA, Soluble MHC class I chain-related protein A; TAMs, Tumor-associated macrophages; TCR, T-cell receptor; tEGFR, Truncated epidermal growth factor receptor; TGF- $\beta$, Transforming growth factor- $\beta$; Th, T helper; TIGIT, Tcell immunoglobulin and ITIM domains; TNFRSF17, Tumor necrosis factor superfamily member 17; Treg, Regulatory T cells; VEGF, Vascular endothelial growth factors; VH, Heavy chain variable domain; VL, Light chain variable domain; XBP1, X-box binding protein 1; Drug abbreviations: Elo-Rd, Elotuzumab with Lenalidomide and Dexamethasone; Rd, Lenalidomide and Dexamethasone; Elo-Pd, Elotuzumab plus Pomalidomide and Dexamethasone; Pd, Pomalidomine and Dexamethasone; Elo-Bd, Elotuzumab plus Bortezomib and Dexamethasone; Bd, Bortezomib and Dexamethasone; Elo-RVd, Elotuzumab plus Lenalidomide (Revlimid), Bortezomib (Velcade), and Dexamethasone; Elo-KRd, Elotuzumab plus Kyprolis (Carfilzomib), Lenalidomide, and Dexamethasone; Elo-PVd, Elotuzumab plus Pomalidomide, Bortezomib, and Dexamethasone; Dara-Rd, Daratumumab with Lenalidomide and Dexamethasone; Dara-Pd, Daratumumab with Pomalidomide and Dexamethasone; Dara-Bd, Daratumumab with Bortezomib and Dexamethasone; Dara-Kd, Daratumumab with Carfilzomib and Dexamethasone; Kd, Carfilzomib and Dexamethasone; Dara-VMP, Daratumumab with Bortezomib, Melphalan and Prednisone; VMP, Bortezomib, Melphalan and Prednisone; Dara-VTd, Daratumumab with Bortezomib plus Thalidomide and Dexamethasone; VTd, Bortezomib plus Thalidomide and Dexamethasone; DaraVRd, Daratumumab with Bortezomib plus Lenalidomide and Dexamethasone; VRd, Bortezomib plus Lenalidomide and Dexamethasone; Isa-Pd, Isatuximab with Pomalidomide and Dexamethasone.
}

antibodies (mAbs) and (iii) agents that stimulate immune cells to selectively kill the malignant cells, such as chimeric antigen receptor (CAR) T-cells, bispecific T-cell engagers (BiTE), and anti-MM vaccines. Those strategies have shown encouraging results in patients with relapsed refractory MM (RRMM) and hold the potential of targeting specifically the malignant cells and the stimulation of a continued response due to harnessing immune surveillance against MM. Nevertheless, the field still presents many challenges, such as the need for tailored therapeutic strategies and biomarkers, the difficulty of selecting the appropriate combination therapy, and resistance to currently available immune-based approaches. Here, we will review the mechanisms that lead to immunosuppression and reduce immune recognition in MM and highlight the strategies created to escape these multiple immune evasion mechanisms to provide long term disease control and better survival for MM patients.

\section{IMMUNE DYSFUNCTION IN MM}

\section{Local Immune Suppression}

Along with the genomic changes occurring in plasma cells (11), the BM microenvironment supports MM progression, development of drug resistance and enable immune escape (6, 12,13 ). It is composed of a cellular compartment (stromal cells, osteoblasts, osteoclasts, endothelial cells, and immune cells), the extracellular matrix components and soluble factors such as cytokines, chemokines, and growth factors. Immune cells are important component of the BM microenvironment. Several functional and numerical defects in the T-cells repertoire have been identified in MM patients. Reduced ratio of CD4:CD8 cells due to a reduction in the total number of $\mathrm{CD}^{+}{ }^{+} \mathrm{T}$-cells is one of the initial defects in parallel with an increase in the number of $\mathrm{CD}^{+}$T-cells (5). Of note, this ratio has been reported to decrease at the time of MM progression, and the reduction of $\mathrm{CD} 4^{+} \mathrm{T}$-cells has been associated with progressed disease and poor prognosis (14). Significantly increased numbers of Thelper (Th) type-1 (Th1), and type-17 (Th-17) have been also noticed in MM patients when compared to patients with monoclonal gammopathy of undetermined significance (MGUS) and healthy donors favoring a suppressive state $(15,16)$. Interleukin (IL)-6 and transforming growth factor- $\beta$ (TGF- $\beta$ ) from the surrounding BM milieu play a critical role in the stimulation of Th- 17 . Th-17 are mainly enhanced in the BM milieu, where they are involved in MM bone disease due to the secretion of IL-17. By cooperating with the receptor activator of nuclear factor kappa-B ligand (RANKL) they can also activate osteoclasts and cause lytic lesions $(17,18)$. Normal in number but defective in their functions also the dendritic cells (DC) have been described to be altered in MM patients. After being stimulated by $\mathrm{CD} 40$ ligand, DCs are incapable of upregulating $\mathrm{B} 7 \mathrm{co}-$ stimulatory molecules such as CD80 and CD86 compromising the antigen presentation to the cytotoxic T-cells and repressing the recognition and killing of MM cells (19). TGF- $\beta$ and IL10 secreted by MM cells have been linked to this deficiency, that can be reverted back by adding IL-12 or interferon- $\gamma$ $(\mathrm{IFN}-\gamma)(20)$. Of note, higher number of plasmacytoid dendritic 
cells (pDCs) have been reported in the BM of MM patients when compared with normal BM. They are knows to have high levels of programmed death 1 ligand (PD-L1) contributing to immune dysfunction and resulting in T-cell inhibition (21, 22). Furthermore, TGF- $\beta$ secreted not only by MM cells, but also by regulatory $\mathrm{T}$ (Treg) cells and $\mathrm{BM}$ stroma cells lead to suppression of the number and function of circulating natural killer (NK) cells, which represent a key cellular subset of the innate immune system (23). The expression of the stimulatory NK receptors NKG2D and the natural cytotoxicity receptor NKp-30 are also reduced in MM patients causing a functional impairment of the NK cells (24). In addition, the secretion by the BM milieu of immune-modulating cytokines such as TGF- $\beta$ and indoleamine 2,3-dioxygenase (IDO) have been also reported to create an immunosuppressive microenvironment. As such increased levels of these factors have been identified in the serum of MM patients $(25,26)$. Secretion of soluble molecules like soluble major histocompatibility complex (MHC) class I chain-related protein A (sMICA) by the malignant MM cells can also facilitate the inhibition of $\mathrm{NK}$ and $\mathrm{CD} 8^{+} \mathrm{T}$-cells by downregulation of NKG2D and it has been linked to poor overall survival (27). Moreover, IDO is an enzyme that catalyzes the rate-limiting first step in the tryptophan catabolism causing tryptophan depletion in the BM milieu and subsequent inhibition of T cells function (28). A summary of the mechanisms leading to MM immune evasion in the BM microenvironment is shown in Figure 1A.

Lastly, an increase in the number of immunosuppressive cells such as Tregs, tumor-associated macrophages (TAMs) and myeloid-derived suppressor cells (MDSCs) have been demonstrated in advanced MM. Tregs express CD25 and transcriptional factor forkhead box P3 (FOXP3) and are capable of inducing expression of co-inhibitory molecules on antigen presenting cells (APCs). They are also known to secrete IL-10 and TGF- $\beta$ and have the capacity to kill APCs and cytotoxic T-cells by using the granzyme- and perforin-dependent pathways (29). In MM TAMs are generally characterized as M2-like macrophages with limited cytotoxicity for tumor cells due to their reduced production of nitric oxide synthases (NOS) and pro-inflammatory cytokines. They have poor antigen-presenting capability and efficiently inhibit Tcell activation (30). Other suppressive cell type increased in MM patients are the MDSCs (31). They are a heterogeneous subset of immature myeloid progenitors cells able to inhibit both innate and adaptive immune responses and stimulate tumor growth (32). As such, they can inhibit T cell functions directly by generating arginases (ARG1), reactive oxygen species (ROS), cyclooxygenase-2 (COX2), inducible NOS (iNOS), and immunosuppressive cytokines (IL-6, IL-10, IL-18), as well as by reducing metabolic factors from the BM microenvironment required for T-cell activation (33). MDSCs have been also reported to inhibit effector $\mathrm{T}$-cell responses by promoting $\mathrm{T}$ reg cell development and by disrupting naive T-cell homing to lymph nodes (34). A summary of the recruitment of immunosuppressive cells in the BM milieu is shown in Figure 1B.

\section{T-Cell Exhaustion}

Tumor-induced impairment of cytotoxic T-cells repertoire at the site of tumor has been also linked to immune escape and failure of immunotherapy-based approaches. An extensively investigated form of T-cell dysfunction is the T-cell exhaustion. Initially described in chronic viral infection and later in cancers, it results from prolonged antigen stimulation and is characterized by gradual loss of T-cells effector activity and increased expression levels of inhibitory receptors (35). To date, the importance of T-cell exhaustion in MM evolution has been ultimately assessed with limited agreement on whether $\mathrm{CD}^{+}{ }^{+}$T-cells can be considered exhausted or senescent. Suen et al. (36) have analyzed the dysfunctional activity of clonal Tcells in MM and demonstrated that immunosenescence is the main feature of these cells. As such, they have showed that the T-cells presented a senescent secretory effector phenotype characterized by positive killer cell lectine like receptor (KLRG$\left.1^{+}\right) / \mathrm{CD} 57^{+} / \mathrm{CD} 160^{+} / \mathrm{CD} 28^{-}$and low programmed death 1 (PD1) and cytotoxic T-lymphocyte-associated antigen 4 (CTLA4) expression, proposing that in MM the T-cells have a molecular signature of senescence rather than exhaustion (36). In addition, Zelle-Rieser et al. (37) described that T-cells from the MM patients were severely more impaired in the BM than in the periphery (peripheral blood). They have demonstrated that at tumor site (BM), the CD8 ${ }^{+}$T-cells expressed several molecules associated with T-cell exhaustion such as PD1 and CTLA-4. In addition, these T-cells lack CD28 and are positive for CD57; a phenotype connected with inferior proliferative capacity and reduced function (37). Of note, Minnie et al. (38) have recently reported on the importance of immune suppression in enabling MM relapse after stem cell transplantation (SCT). They have demonstrated that BM-infiltrating $\mathrm{CD}^{+}$T-cells from MMrelapsed mice are phenotypically and functionally exhausted in RRMM patients following SCT. This was associated with increased production of IL-10 and increased expression of T-cell immunoglobulin and immunoreceptor tyrosine-based inhibition motif (ITIM) domains (TIGIT) and PD1 (38), highlighting the importance of early use of checkpoint inhibitions in MM to avoid T-cell exhaustion and provide a long-term immunological control.

\section{STRATEGIES TO RESTORE IMMUNE RESPONSES IN MYELOMA}

\section{Agents That Remove the Breaks of the Immune System \\ Immunomodulatory Agents}

Immunosuppression plays a critical role in MM pathogenesis, therefore reversing this suppression could potentially reinstate MM immune surveillance and improve disease control. IMiDs exhibit potent anti-MM activity (39-41). They possess pleiotropic properties including cytotoxic and immunomodulatory effects (42). In MM cells, through binding to cereblon (CRBN), an adaptor protein of the Cul4A-DDB1-ROC1 ubiquitin E3 ligase complex, they induce proteasomal degradation of the 


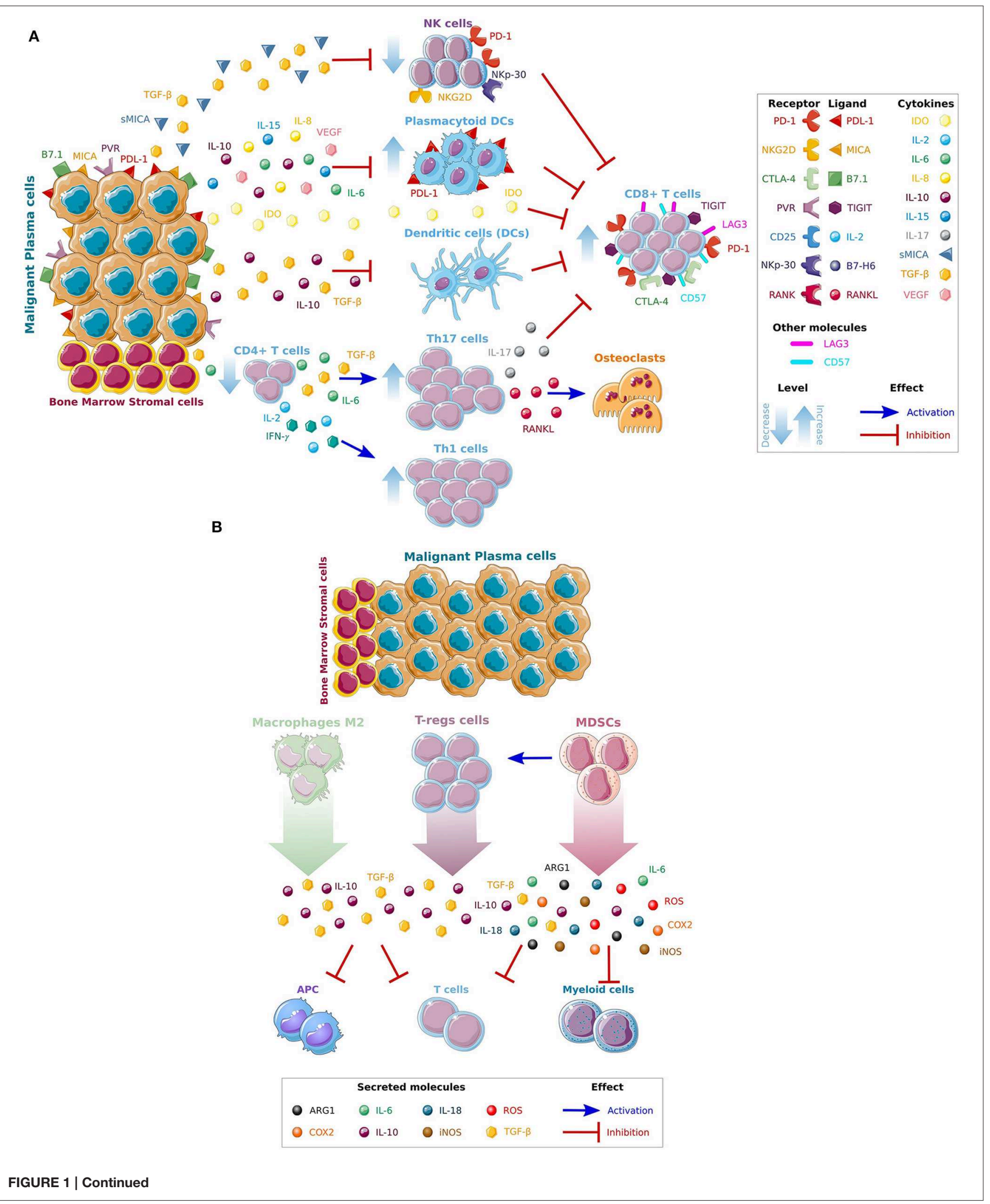


FIGURE 1 | Mechanisms leading to MM immune escape. (A) Direct and indirect effects of MM cells on immune cells. The complex interactions of BM stromal cells and tumor cells, through the production of different cytokines and growth factors (IL6, IL8, IL10, IL15, VEGF) and immune inhibitors factors (sMICA,TGF- $\beta$, and IDO) released by MM cells, promotes MM growth and inhibit the activity of cytotoxic T (CD8+), dendritic (DC), and natural killer (NK) cells. (B) Recruitment of immunosuppressive cells in the BM microenvironment. An immunosuppressive microenvironment is also maintained by the recruitment of immunosuppressive cells such as macrophages M2, T-regs and Myeloid-derived suppressor cells (MDSCs) that further facilitate immune escape and promote disease progression. Interleukins IL-6, IL-8, IL-10, and IL-15, the vascular endothelial growth factor (VEGF), soluble major histocompatibility complex (MHC) class I chain-related protein A (sMICA), transforming growth factor beta (TGF- $\beta$ ), indoleamine 2,3-dioxygenase (IDO), T-helper (Th-), programmed death 1 (PD-1), cytotoxic T-lymphocyte-associated antigen 4 (CTLA-4), T-cell immunoglobulin and ITIM domains (TIGIT), lymphocyte-activation gene 3 (LAG-3), antigen presenting cells (APC), reactive oxygen species (ROS), cyclooxygenase-2 (COX2), and inducible nitric oxide synthase (iNOS). *Figures have been created using Smart Servier Medical Art website.

lymphoid transcription factors IKAROS family zinc finger 1 (IKZF1), AIOLOS (IKZF3) $(43,44)$ and casein kinase $1 \alpha(\mathrm{CK} 1 \alpha)$ (45) leading to the transcriptional repression of the interferon regulatory factor 4 (IRF4) and MYC. In an IKZF3 dependent mechanism, IMiDs stimulate T-cell proliferation and induce IL-2 and IFN $\gamma$ secretion $(44,46)$. These cytokines increase the number of NK cells and improve their function to facilitate lysis of MM cells (47). IMiDs are also known to decrease the activity of Tregs (48) and enhance cytotoxic T lymphocyte (CTL, CD8 ${ }^{+}$ $\mathrm{T}$ cells) activity against MM cells $(49,50)$. Furthermore, they improve tumor antigen uptake by DCs improving the efficacy of antigen presentation (51). These properties mark IMiDs as attractive backbone to use in combination with other anti-MM therapies, in particular the immune-based strategies. As such, in combination with monoclonal antibodies such as Elotuzumab targeting the glycoprotein SLAM family member 7 (SLAMF7), also known as CS1 $(52,53)$ or Daratumumab and Isatuximab targeting the $\mathrm{CD} 38$, IMiDs have showed significant synergistic effects, increase in overall response rate (ORR) and extension of progression-free survival (PFS) and overall survival (OS) in MM patients (54-56).

Of note, the synergistic phenomena reported when antiCD38 antibodies are combined with IMiDs seem to derive from their co-modulated effects on the host adaptive and innate immunity, suggesting that the acquired resistance to this combination may be mainly immune-mediated. These CD38 targeting antibodies have been reported by many groups (including ours) to exert multiple anti-tumoral immune effect such as complement dependent cytotoxicity (CDC), antibody mediated cellular phagocytosis (ADCP) as well as antibody driven cellular cytotoxicity (ADCC) $(57,58)$. Previous work from our laboratory (59) has also demonstrated that in responding patients Daratumumab induces expansion of Tcells and increases T-cell clonality. In contrast, an increase of exhausted T-cells with upregulation of the checkpoint inhibitors lymphocyte-activation gene 3 (LAG3) and TIGIT was observed in resistant patients. Those findings identified T-cell exhaustion as cellular mediator of resistance to anti-CD38 antibodies and warrant further investigation of LAG3- and/or TIGITblocking approaches as possible ways to reinstate sensitivity to Daratumumab.

\section{Immune Checkpoint Blockade}

Co-stimulatory and co-inhibitory immune checkpoints tightly regulate the immune response upon activation to defend the host from autoimmunity or harm due to inflammation (60). Tumor cells are known to evade immune surveillance by upregulating ligands for inhibitory immune receptors triggering an exhaustion profile of T-cells (61). Efforts to reverse this exhausted phenotype have been achieved by blocking the inhibitors receptors expressed on T-cells though an immune checkpoint blockade. Instead of working directly on the tumor cells, these agents excite the host's immune system to induce an anti-tumor effect (62). Clinically, the most important checkpoints to date are CTLA-4 and PD-1/PDL1 axis that have proven to be active in various solid tumors and hematologic malignancies $(63,64)$. In MM, although MM cells express PDL-1, the BM cytotoxic T-cells have low levels of $\mathrm{PD}-1$, suggesting that $\mathrm{PD}-1$ blockade may not be adequate to stimulate T-cells (65). Blocking antibodies targeting PD1 (Pembrolizumab and Nivolumab) and PD-L1 (Durvalumab) have been assessed in MM. In monotherapy they did not show clinical responses, probably due to the described immune dysfunction reported in MM $(66,67)$. Better responses have been demonstrated in combination with IMiDs, due to the potential synergistic activation of adaptive and innate immunity (68, 69). However, those combinations were associated with various adverse events (AEs) (pulmonary, cardiac, gastrointestinal and hepatic toxicity) and an increased risk of death raising safety concerns. Therefore, while immune checkpoint inhibitors remain an attractive therapy for MM patients, further studies are required to increase clinical activity and limit the immunemediated toxicity. The anti-PD-1 Cemiplimab is currently being evaluated in a phase I-II trial in combination with the antiCD38 Isatuximab, whereas Durvalumab is under evaluation in combination with the other anti-CD38 Daratumumab. In addition, other checkpoint inhibitors (LAG-3, TIGIT, TIM3 and $\mathrm{CD} 85 \mathrm{j}$ ) have been demonstrated relevant during $\mathrm{MM}$ progression (70-73) and antibodies against theses checkpoints inhibitors are in initial stages of development. A summary of combination trials with checkpoint inhibitors ongoing in MM is presented in Table 1.

Furthermore, since epigenetic abnormalities have been observed in cancer cells and tumor infiltrating T-cells (74) epigenetic modulating agents could be used to enhance antitumor immunity. DNA methyltransferase and inhibitors of histone deacetylase have been described to modify anti-tumor immune responses in numerous cancers $(75,76)$ including $M M$ (77-79). Therefore, the potential synergy between epigenetic and immune therapies could also be further explored. 
TABLE 1 | Summary of combination trials with checkpoint inhibitors ongoing in MM.

\begin{tabular}{|c|c|c|c|c|c|}
\hline Target & Type of therapy & Compound & Combination & Phase & NCT number \\
\hline PD-1 & Immune checkpoint blockade & Cemiplimab & Isatuximab & $|-| \mid$ & NCT03194867 \\
\hline PD-1 & Immune checkpoint blockade & Nivolumab & Pomalidomide and Dexamethasone & III & NCT02726581 \\
\hline PD-1 & Immune checkpoint blockade & Nivolumab & Lenalidomide & $\|$ & NCT03333746 \\
\hline PD-1 & Immune checkpoint blockade & Nivolumab & Daratumumab and Cyclophosphamide & $\|$ & NCT03184194 \\
\hline PD-1 & Immune checkpoint blockade & Nivolumab & Dexamethasone, Carfilzomib, Nivoluman, and Reovirus & I & NCT03605719 \\
\hline PD-1 & Immune checkpoint blockade & Pembrolizumab & Pomalidomide and Dexamethasone & $|-| \mid$ & NCT02289222 \\
\hline PD-1 & Immune checkpoint blockade & Pembrolizumab & Lenalidomide and Dexamethasone & 1 & NCT02036502 \\
\hline PDL-1 & Immune checkpoint blockade & Durvalumab & Daratumumab & I & NCT03000452 \\
\hline
\end{tabular}

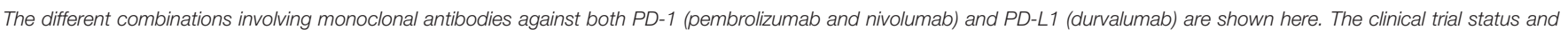
numbers are indicated here.

\section{Agents That Target Highly Selective Antigens on the MM Cells in the Form of Monoclonal Antibodies}

Monoclonal antibodies (mAbs) have recently emerged as active therapeutic agents for the management of MM patients. They target highly selective antigens expressed in malignant plasma cells and not in normal tissues, stimulating specific anti-tumor activity and preventing toxicity due to off target effects. They elicit anti-MM activity through multiple mechanisms, including a direct cytotoxic effect on MM cells via apoptosis and an immune-mediated cytotoxicity such as ADCC, CDC, and ADCP. They can also be used to directly target the malignant plasma cells while releasing an anti-cancer agents linked via a chemical linker, as it is the case for the antibody-drug conjugates (ADCs), or to engage and stimulate cytotoxic T-cells for lysis of MM cells with bispecific T-cell engagers (BiTEs).

\section{Anti-CS1/SLAMF7 Monoclonal Antibody}

Elotuzumab is a humanized immunoglobulin (Ig) $\mathrm{G}_{1}$ monoclonal antibody that targets SLAMF7, also known as CS1, a glycoprotein greatly expressed in MM cells and NK cells (80). It mediates the killing of MM cells by NK cellassociated ADCC, NK cell activation and by inhibiting the interactions between MM cells and BM stromal cells (BMSCs) (81). Encouraging results were observed in RRMM patients treated with Elotuzumab when combined with IMiDs such as Lenalidomide and Pomalidomide $(53,82)$. The combination of Elotuzumab with Lenalidomide and Dexamethasone (Elo-Rd) were assessed in a phase III trial (ELOQUENT-2). In this trial, the triple regimen containing Elotuzumab demonstrated to be clinically superior than Lenalidomide and Dexamethasone (Rd) in terms of ORRs (79 vs. 66\%), PFS (19.4 months vs. 14.9 months) and OS (48 vs. 40 months) without additional toxicity $(53,83)$. The separation of OS curves was also maintained over time in favor of Elo-Rd with 4 years OS rate of 50 vs. $43 \%$ for $\mathrm{Rd}$ (84). Elo-Rd is currently approved by both the Food and Drug Administration (FDA) and European Medicines Agency (EMA) for the treatment of RRMM patients after one line of prior therapy. A phase II trial of Elotuzumab in combination with Pomalidomide and Dexamethasone (Elo-Pd) vs. Pomalidomine and Dexamethasone (Pd) (ELOQUENT-3) in patients who received more than two previous therapies demonstrated that after a follow-up period of 9 months, Elo-Pd had a longer PFS (10.3 vs. 4.7 months) and a better ORR (53 vs. $26 \%$ ) as compared to Pd alone (52). Furthermore, the Phase II trial of Elotuzumab plus Bortezomib and Dexamethasone (Elo-Bd) vs. Bortezomib and Dexamethasone (Bd) in patients who received from one to three prior therapies showed that Elo-Bd is also associated with longer median PFS (9.7 vs. 6.9 months). However, there was still no differences in ORR between these two groups (66 vs. 63\%) $(85,86)$. Several studies are now ongoing to evaluate Elotuzumab-based combinations, such as Elotuzumab plus Lenalidomide, Bortezomib, and Dexamethasone (Elo-RVd), Elotuzumab plus Carfilzomib, Lenalidomide, and Dexamethasone (Elo-KRd) and Elotuzumab plus Pomalidomide, Bortezomib, and Dexamethasone (EloPVd). A summary of combination trials with anti-CS1/SLAMF7 ongoing in MM is shown in Table 2.

\section{Anti-CD38 Monoclonal Antibodies}

CD38 is a transmembrane glycoprotein highly expressed in MM cells and at low level in plasma, myeloid, and lymphoid cells, and some non-hematopoietic tissues (87). It has been reported to have ectoenzymatic activity and several functions in cell adhesion, signal transduction and calcium signaling $(88,89)$.

Daratumumab is the first anti-CD38 targeting antibody approved as monotherapy and in combination with numerous anti-MM standard regiments in MM. It is a fully humanized $\mathrm{IgG}_{1}$ карра monoclonal antibody that targets the cyclic ADP ribose hydrolase CD38. It mediates the killing of $\mathrm{MM}$ cells via $\mathrm{CDC}, \mathrm{ADCC}, \mathrm{ADCP}$, and direct apoptosis via FcRmediated cross-linking, and modulation of CD38 enzyme activities $(57,58,90)$. Additionally, Daratumumab has showed an immunomodulatory role by promoting $\mathrm{CD}_{3} 8^{+}$immune regulatory cell and stimulating $\mathrm{T}$-cell expansion. This process is associated with the increase of helper and cytotoxic T-cells, T-cell functional responses, and T-cell receptor (TCR) clonal expansion (91). Clinically, it demonstrated anti-MM activity both as monotherapy and when combined with novel agents in heavily pretreated RRMM patients. As such, the phase I GEN501 and phase II SIRIUS trials demonstrated that Daratumumab is effective as single agent in heavily pretreated patients and showed 
TABLE 2 | Summary of combination trials with monoclonal antibodies ongoing in MM.

\begin{tabular}{|c|c|c|c|c|c|c|}
\hline Target & Type of therapy & Compound & Combination & Phase & NCT number & Trial name \\
\hline SLAMF7 & Monoclonal antibody & Elotuzumab & Lenalidomideand Dexamethasone & III & NCT01239797 & ELOQUENT-2 \\
\hline SLAMF7 & Monoclonal antibody & Elotuzumab & Pomalidomide and Dexamethasone & $\|$ & NCT02654132 & ELOQUENT-3 \\
\hline SLAMF7 & Monoclonal antibody & Elotuzumab & Bortezomib and Dexamethasone & $\|$ & NCT01478048 & \\
\hline SLAMF7 & Monoclonal antibody & Elotuzumab & Lenalidomide, Bortezomib, and Dexamethasone & $\|$ & NCT02375555 & \\
\hline SLAMF7 & Monoclonal antibody & Elotuzumab & Kyprolis, Lenalidomide, and Dexamethasone & $\|$ & NCT02969837 & \\
\hline SLAMF7 & Monoclonal antibody & Elotuzumab & Pomalidomide, Bortezomib, and Dexamethasone & $\|$ & NCT02718833 & \\
\hline CD38 & Monoclonal antibody & Daratumumab & Lenalidomide and dexamethasone & III & NCT02076009 & POLLUX \\
\hline CD38 & Monoclonal antibody & Daratumumab & Pomalidomide and Dexamethasone & $\|$ & NCT01998971 & EQUULEUS \\
\hline CD38 & Monoclonal antibody & Daratumumab & Pomalidomide and Dexamethasone & III & NCT03180736 & APOLLO \\
\hline CD38 & Monoclonal antibody & Daratumumab & Bortezomib and Dexamethasone & III & NCT02136134 & CASTOR \\
\hline CD38 & Monoclonal antibody & Daratumumab & Carfilzomib and Dexamethasone & III & NCT03158688 & CANDOR \\
\hline CD38 & Monoclonal antibody & Daratumumab & Bortezomib, Melphalan, and Prednisone & III & NCT02195479 & ALCYONE \\
\hline CD38 & Monoclonal antibody & Daratumumab & Lenalidomide and Dexamethasone & III & NCT02252172 & MAIA \\
\hline CD38 & Monoclonal antibody & Daratumumab & Bortezomib, Thalidomide, and Dexamethasone & III & NCT02541383 & CASSIOPEIA \\
\hline CD38 & Monoclonal antibody & Daratumumab & Bortezomib, Lenalidomide, and Dexamethasone & $\|$ & NCT02874742 & GRIFFIN \\
\hline CD38 & Monoclonal antibody & Isatuximab & Pomalidomide and Dexamethasone & III & NCT02990338 & ICARIA \\
\hline CD38 & Monoclonal antibody & Isatuximab & Kyprolis and Dexamethasone & III & NCT03275285 & IKEMA \\
\hline CD38 & Monoclonal antibody & MOR202 & & 1 & NCT01421186 & \\
\hline CD38 & Monoclonal antibody & TAK-079 & & 1 & NCT03439280 & \\
\hline
\end{tabular}

The different combinations involving anti-SLAMF7 and anti-CD38 mAbs are shown here. The clinical trial status and numbers are indicated here.

improved ORRs (29\%), median PFS (mPFS) and OS of 3.7 and 17.5 months respectively (92-94). Based on these results FDA and EMA have now approved Daratumumab as single agent for RRMM patients who have received at least three prior lines of therapy including a proteasome inhibitors (PIs) and an IMiD.

In combination with Lenalidomide and Dexamethasone, Daratumumab (Dara-Rd) demonstrated significant efficacy in the phase III POLLUX trial. The ORR was $92.9 \%$ in Dara$\mathrm{Rd}$ patients vs. 72.9 in $\mathrm{Rd}$ group, with a mPFS not reached vs. 17.5 months in the Dara-Rd vs. Rd arm and higher rate of patients achieving deep response with a minimal residual disease (MRD) negativity of $26 \%$ in the DRd group vs. $6 \%$ in the $\mathrm{Rd}$ group $(54,55,95)$. Based on these data Dara-Rd is now approved for the treatment of MM patients who have previously received at least one line of therapy. In addition, Daratumumab has been used in combination with Pomalidomide and Dexamethasone (Dara-Pd). In the phase II trial EQUULEUS the three drug regimen showed an ORR of $60 \%$, a mPFS and OS of 8.8 and 17.5 months respectively in heavily pretreated patients (96). Conclusive results will be derived from the ongoing phase III trial APOLLO, deigned to compare Dara-Pd vs. Pd in RRMM patients.

Daratumumab has been also combined with PIs. The phase III CASTOR trial revealed that adding Daratumumab to Bortezomib and Dexamethasone (Dara-Bd) resulted in higher ORR (83 vs. 63\%), extended PFS (median 16.7 vs. 7.1 months) and higher MRD negativity rate (12 vs. 2\%) (97-99). Based on these data the triplet Dara-Bd is also currently approved by the FDA and EMA for RRMM patients. In combination with Carfilzomib and Dexamethasone (Dara-Kd), a phase Ib showed that Daratumumab induced a clinical response in $84 \%$ of RRMM patients, previously receiving Lenalidomide and Bortezomib (100). Of note, a recent report on the ongoing phase III CANDOR study comparing Dara-Kd vs. Carfilzomib and Dexamethasone $(\mathrm{Kd})$ demonstrated prolonged PFS with Dara-Kd vs. Kd (not reached vs. 15.8 months), ORR of 84.3 vs. $74.7 \%$ and higher MRD negativity rate (12.5 vs. $1.3 \%)$ (101). Based on these encouraging results Daratumumab is rapidly moving toward the first line treatment. As such, in older patients with newly diagnosed MM (NDMM) results from the phase III ALCYONE trial showed that Daratumumab in combination with Bortezomib, Melphalan and Prednisone (Dara-VMP) had impressive responses with an ORR of $91 \%$ vs. $74 \%$ with Bortezomib, Melphalan and Prednisone (VMP). At a median follow-up of 17 months an extended mPFS (not reached vs. 18.1 months) and increased MRD rate (22 vs. $6 \%$ ) was reported with Dara-VMP as compared to VMP (99, 102). At median follow-up of 28 months, remarkable results in terms of higher MRD negativity (24.2 vs. $7.3 \%$ ) and lower risk of progression or death (mPFS not reached vs. 32 months) were also observed in the phase III MAIA trial when Dara-Rd was compared to $\mathrm{Rd}$ in NDMM patients transplant-ineligible (95, 103). In NDMM patients eligible for Autologous SCT (ASCT), Daratumumab has also been used in combination with standard triplets such as Bortezomib plus Thalidomide and Dexamethasone (VTd), Bortezomib plus Lenalidomide and Dexamethasone (VRd), Dara-VTd and Dara-VRd. In the phase III CASSIOPEIA trial, following consolidation, the rate of MRD negativity was higher in the Dara-VTd group than in the VTd group (64 vs. 44\%). These responses translated into a 53\% reduction in the risk of progression or death for the DaraVTd group vs. the VTd group (104) and led to the FDA 
approval of Dara-VTd as induction therapy for transplant eligible patients. VRd in combination with Daratumumab (Dara-VRd) has been also evaluated in the ongoing phase II trial GRIFFIN. Results presented at the last American Society of Hematology (ASH) 2019 meeting showed that the addition of Dara to VRd led to significant improvement in stringent complete remission (sCR) and depth of response when compared to VRd alone and did not affect the stem cell mobilization or hematopoietic reconstitution (105).

Isatuximab is a chimeric $\operatorname{IgG}_{1-\kappa}$ anti-CD38 $\mathrm{mAb}$ which selectively targets a unique epitope on human $\mathrm{CD} 38$ receptor and induces anti-MM activity by direct apoptosis, CDC, ADCC, and ADCP (106). Similarly to Daratumumab, Isatuximab demonstrated promising clinical activity in heavily pre-treated MM patients as single agent (107) and when used in combination with different anti-MM agents. In a phase Ib trial Isatuximab in combination with $\mathrm{Rd}$ showed an ORR of $51 \%$ and mPFS of 8.5 months in heavily pretreated patients of whom $68 \%$ had already received Carfilzomib or Pomalidomide (108). In combination with Pomalidomide and Dexamethasone the ORR was $62 \%$ and $\mathrm{mPF}$ was 17.6 months (109). The phase III ICARIA trial comparing the triplet Isatuximab-Pomalidomide-Dexamethasone (Isa-Pd) to the duplet Pomalidomide-Dexamethasone (Pd) in RRMM patients receiving at least two previous lines of therapy is presently ongoing. Recently, a constant advantage in terms of ORR (60 vs. $35 \%$ ) and mPFS (11.5 months vs. 6.5$)$ was reported in the Isa-Pd group compared to the control group $\mathrm{Pd}$ at a median follow-up of 11.6 months, (56). The phase III IKEMA trial evaluating the combination of Isatuximab with Kd in RRMM patients is now ongoing and results awaited (110).

Of note, others anti-CD38 antibodies currently being evaluated include MOR202 (fully human from Morphosys), and TAK-079 (fully human from Takeda). Clinical activities in RRMM patients have been reported in combination with IMiDs $(111,112)$ and phase III trials are expected to start soon. A summary of combination trials with anti-CD38 mAbs ongoing in $\mathrm{MM}$ is presented in Table 2.

\section{Agents That Stimulate Immune Cells to Selectively Kill the Malignant Cells Chimeric Antigen Receptor (CAR) - T Cell}

In recent years, the development of therapeutic agents able to induce the autologous immune cells to mediate tumor cell killing and to overcome the immunosuppressive mechanisms of the tumor microenvironment has revolutionized the treatment of cancers. In this setting adaptive cell therapy using chimeric antigen receptor (CAR)-T cell therapy has been developed to eliminate cancer cells in many hematological malignancies including MM. CARs are artificial fusion proteins that consist of the extracellular antigen recognition part of an antibody from a single-chain variable fragment ( $\mathrm{scFv}$ ) fused with the $\mathrm{CD} 3 \xi$ chain for the intracellular signaling, and a $\mathrm{T}$ cell costimulatory domains (CD28 or $4-1 \mathrm{BBB})$ (113). Via the scFv, CAR-transduced T-cells (CAR-T) can directly recognize and bind the antigen of interest, while the intracellular domain composed of the $\mathrm{Cd} 3 \zeta$ chain of the
T-cell receptor (TCR) induces T-cell activation (114). In contrast to a TCR, CAR-T cells are not restricted by MHC class and can recognize the antigen express on target cells independently of the MHC haplotype and the antigen presenting machinery.

Different antigen have been tested as targets for CAR-T cell therapy against MM. These include CD44v6, CD70, CD38, CD138, SLAMF7, and class C group 5 member D (GPRC5D) $(115,116)$. An important factor in the development of a successful CAR is selecting a proper surface antigen target that is absent in normal cells. To date the most promising antigen target is the B-cell maturation antigen (BCMA), and in this section we will focused on the different CAR-T cell therapies targeted this antigen in MM patients.

BCMA, a transmembrane signaling protein member of the tumor necrosis factor superfamily member 17 (TNFRSF17 or CD269), is expressed in mature B lymphocytes, and is important in maintaining the long-lived plasma cell homeostasis (117). It is also uniformly expressed in malignant plasma cells. Gammasecretase directly shed BCMA from plasma cells (118), resulting in a soluble form that can be identified in peripheral blood where its serum levels correlate with response to therapy and overall survival (119). It binds to two cognate ligands B-Cell Activating Factor (BAFF) and A Proliferation Inducing Ligand (APRIL) leading to NF-kappaB and MAPK8/JNK activation and delivering critical survival signals for MM cells (120). As such, numerous anti-BCMA CAR-T cell therapies have been developed and showed impressive clinical activities in RRMM patients.

The first in-human trial using an anti-BCMA CAR-T cells was completed at the National Cancer Institute (NCI) by Brudno et al. (121). They have used a retrovirally transduced second generation of CAR generated by using a CD28 costimulatory domain and a murine scFv. At the highest dose the authors reported an ORR of $81 \%$, with at least a complete response (CR) achieved in $13 \%$ of patients. Clinical responses and depth of response were positively correlated with the expansion of $\mathrm{CAR}^{+} \mathrm{T}$-cell peak. Of note, cytokine release syndrome (CRS) of grade $(\mathrm{G})$ 3-4 was not observed in the dose-expansion phase (121). Next, Cohen et al. (122) from the University of Pennsylvania in Philadelphia reported the clinical results of the phase I study conducted by using a fully human lentivirally transduced anti-BCMA CAR with the 4-1BB costimulatory domain. In the three cohorts enrolled on the study, the ORR was $44 \%$ in cohort $1,20 \%$ in cohort 2 , and $64 \%$ in cohort 3 and the mPFS was 2.2, 1.9, and 4.2 months respectively (122). $\mathrm{Bb} 2121$ is the second generation of anti-BCMA-CAR expressing the same $\mathrm{scF}$ c portion as the NCI trial with a $4-1 \mathrm{BB}$ costimulatory domain. Results from the multicenter phase I dose escalation trial testing bb2121 in RRMM have been recently reported and demonstrated an ORR of $85 \%$ with $45 \%$ of patients in CR or better. The clinical responses were quick with a median time to response of one month and all responding patients were MRDnegative. The median duration of response was 10.9 months and the mPFS was 11.8 months (123). The phase II single-arm trial evaluating the efficacy and safety of bb2121 in RRMM patients (KarMMa) has just finished enrollment and results awaited.

Following bb2121, the phase I trial of the next generation of anti-BCMA CAR-T therapy bb21217 has been also reported. The 
TABLE 3 | Summary of combination trials with anti-BCMA T-cell therapies ongoing in MM.

\begin{tabular}{llllcl}
\hline Target & $\begin{array}{l}\text { Type of } \\
\text { therapy }\end{array}$ & Compound & Combination & Phase NCT number \\
\hline BCMA & CAR-T cell & Bb2121 & I & NCT02658929 \\
BCMA & CAR-T cell & Bb2121 & II & NCT03361748 \\
BCMA & CAR-T cell & Bb21217 & I & NCT03274219 \\
BCMA & CAR-T cell & LCAR-B38M & I & NCT03090659 \\
BCMA & CAR-T cell & LCAR-B38M & Ib-I & NCT03548207 \\
BCMA & CAR-T cell & JCARH125 & I-I & NCT03430011 \\
BCMA & CAR-T cell & MCARH171 & I & NCT03070327 \\
BCMA & CAR-T cell & FCARH143 & I & NCT03338972 \\
BCMA & ADCs & GSK2857916 & Pembrolizumab & II & NCT03848845 \\
BCMA & ADCs & GSK2857916 & Pomalidomide & I-I & NCT03715478 \\
BCMA & ADCs & GSK2857916 & Lenalidomide/ & II & NCT03544281 \\
& & & Borthezomib and & & \\
& & & Dexamethasone & & \\
BCMA & BiTE & AMG 420 & & I & NCT02514239 \\
BCMA & BiTE & AMG 701 & I & NCT03287908 \\
\hline
\end{tabular}

The different trials with anti-BCMA T-cell therapies (CAR-T and BiTES) ongoing in MM are presented here. The clinical trial status and numbers are shown here.

use of the phosphoinositide 3 kinase (PI3K) inhibitor bb007, to increase of memory-like CAR-T cells the final product, indicated similar ORR and toxicity profile to what was observed with bb2121. A longer follow-up and clinical data from patients receiving higher doses of cells are now required to understand if the ex vivo manipulation of the $\mathrm{T}$ cell products will result in enhanced efficacy (124).

To improve the effects of anti-BCMA CAR-T therapy, a CART cell therapy targeting two different BCMA epitopes (VHH1 and VHH2) was recently developed (LCAR-B38M). It showed a high response rate, with an ORR of $88 \%$, an MRD negativity of $63 \%$ and a median PFS of 15 months in RRMM patients $(125,126)$. Based on these clinical results, a phase Ib-II trial CARTITUDE-1 is currently ongoing in RRMM patients.

Additional CAR-T clinical trials targeting BCMA also include the JCARH125, MCARH171, and FCARH143 studies. They use three new CAR-T cell products composed of a human-derived scFv, a 4-1BB costimulatory domain, and a truncated human epidermal growth factor receptor (tEGFR), respectively, and are currently being evaluated in phase I clinical trials. A summary of combination trials with anti-BCMA CAR-T cell therapies ongoing in MM is presented in Table 3.

In addition to CAR-T therapy BCMA is also a perfect target for antibody-drug conjugates (ADCs) and for bispecific T-cell engagers (BiTEs). ADCs are immunoconjugates composed of a monoclonal antibody conjugated to a cytotoxic drug via a chemical linker. They precisely target cells expressing the target antigen and are then internalized to release the cytotoxic component and lead to cell death (127). Clinical results of the novel anti-BCMA-ADC conjugated to the antimitotic agent monomethyl auristatin F (GSK2857916) were recently reported. In heavily pre-treated MM patients, GSK285791 showed an ORR in $60 \%$ of patients with a mPFS of 12 months and 7.9 in patients refractory to both IMiDs and PIs (128). Additional trials are now evaluating its safety and efficacy in combination with Pembrolizumab, Pomalidomide, and Lenalidomide or Bortezomib in RRMM patients (Table 3).

\section{Bispecific T-Cell Engagers}

BiTEs are engineered molecules able to direct the host's immune system, more precisely the T-cells, against cancer cells. They are recombinant bispecific proteins with two linked scFvs. from two different antibodies, one targeting a cell-surface molecule on $\mathrm{T}$ cells (e.g., $\mathrm{CD} 3 \varepsilon$ ) and the other targeting antigens on the surface of malignant cells. By binding to tumor antigens and T-cells simultaneously, BiTEs mediate Tcell responses and killing of tumor cells (129). Advantages of BiTEs include their ability to function independently of $\mathrm{MHC}$ haplotype and co-stimulation, and do not require peptide antigen presentation $(130,131)$. Furthermore, these molecules do not need ex vivo manipulation of $\mathrm{T}$-cell and have relative simple production and purification allowing immediate treatment. The first approved BiTE was Blinatumomab, an anti-CD19, used for the treatment of relapse/refractory B cell acute lymphoblastic leukemia (ALL) (132).

In MM, among potential targets, BCMA, CD38, SMALF7, FcRH5, and G protein-coupled receptor (GPCR) GPRC5D, have been selected to develop anti-MM BiTEs, with BCMA representing the most promising target. As such, AMG 420 is the first anti-BCMA BiTE currently being evaluated in MM. It contains the scFv targeting BCMA in its $\mathrm{N}$-terminal and CD3 $\xi$ in its C-terminal followed by a hexa-histidine (His6-tag) (133). Clinical results of the first-in-human dose escalation trial in RRMM patients, progressed after more than two lines of therapy, were recently presented. In this study AMG 420 induced an ORR of $70 \%$, including $50 \%$ MRD-negative complete responses at the maximum tolerated dose (MTD) (134). A longer follow-up and more mature data are now needed to understand whether or not BiTEs will improve efficacy when compared to CAR-T therapy. Another anti-BCMA BiTE with an extended half-life and weekly short infusion, AMG 701, has showed significant activity in preclinical studies (135) and is currently being investigated in a phase I trial.

Research investigating tri-specific antibodies is also emerging. As such, HPN217 is the first in this category. It is designed to recognize human BCMA to target $\mathrm{MM}$ cells, serum albumin to extend its half-life, and $\mathrm{CD} 3 \varepsilon$ for the engagement of $\mathrm{T}$ cells. Preclinical studies have demonstrated BCMA- and T celldependent anti-tumor activity in vitro and in xenografts models of MM and lymphoma (136) and is currently under evaluation for further development and commercialization. A summary of combination trials with anti-BCMA BiTEs ongoing in MM is presented in Table 3.

\section{Anti-MM Vaccination Approaches}

Anti-cancer vaccines are based on the use of tumor antigens to stimulate the immune system and produce an antitumor response. To date, several therapeutic vaccine strategies have been established. These include the use of whole tumor cell, gene-modified tumor cells, or tumor-cell lysates, peptide or 
protein-based vaccines, RNA- and DNA- based vaccines, viral vector modified to express tumor antigen and DC-based vaccines containing DNA, RNA, or peptides $(137,138)$.

Numerous preclinical studies and clinical trials using these diverse therapeutic strategies have been completed and reported to be promising for the treatment of indolent metastatic disease $(139,140)$.

In $\mathrm{MM}$ these approaches have been used in disease stages with lower tumor burden including stem cell transplantation (SCT), precursor disease such as smoldering myeloma (SMM), and MRD settings (141). In the setting of transplantation studies have evaluated vaccines targeting hTERT, MAGE-A3, or survivin in combination with vaccine-primed autologous lymphocyte infusion (142-144). In SMM a multi-peptide vaccine PVX-140 has been designed to induce a $\mathrm{T}$ cell mediated immune response by specifically stimulating CTLs with the tumor antigen targets X-box binding protein 1 (XBP1), Syndecan-1 (CD138), and SLAMF7 (CS1). Memory CD8 ${ }^{+}$T-cell responses were reported and the vaccine demonstrated single-agent immunogenicity that was enhanced by the addition of Lenalidomide (145).

Among the cell-based vaccines, therapeutic strategies based on the use of autologous DCs pulsed with tumor antigens have been tested. As such, in a phase II trial, a fusion vaccine generated by combining autologous MM and DCs was administrated to MM patients following ASCT (146). It demonstrated a myeloma specific CD4 and CD8 T-cell responses with $24 \%$ of patients achieving a partial response (PR), that following vaccination, were converted to complete response $(\mathrm{CR}) /$ near $\mathrm{CR}$ (nCR), due to the effect of the vaccine on remaining disease (146). A clinical trial evaluating this fusion vaccine combined with a PD-1 inhibitor is now ongoing.

Overall, anti-MM vaccination therapy appears to be well tolerated and largely considered to have the greatest activity when used in combination with other therapies that have immunomodulatory properties. In this context, vaccines could increase the probability of clinical response or improve its duration making this approach a promising adjuvant strategy against MM.

\section{DISCUSSION}

The treatment of MM patients has improved significantly over the past few years (147). A better understanding of the immuneescape mechanisms contributing to tumor progression, has led to the development of several active and well-tolerated forms of

\section{REFERENCES}

1. Kazandjian D. Multiple myeloma epidemiology and survival: a unique malignancy. Semin Oncol. (2016) 43:676-81. doi: 10.1053/j.seminoncol. 2016.11.004

2. Cowan AJ, Allen C, Barac A, Basaleem H, Bensenor I, Curado MP, et al. Global Burden of Multiple Myeloma: A Systematic Analysis for the Global Burden of Disease Study 2016. JAMA Oncol. (2018) 4:1221-7. doi: 10.1001/jamaoncol.2018.2128

3. Palumbo A, Anderson K. Multiple myeloma. N Engl J Med. (2011) 364:104660. doi: 10.1056/NEJMra1011442 immunotherapy that has significantly improved outcome of MM patients $(9,10)$.

IMiDs with their pleiotropic anti-MM properties have showed ability to enhance the effects of mAb treatments, checkpoint inhibitors and $\operatorname{ADCs}(55,69,84,128)$. Other antibody-based immunotherapies, such as CAR-T and BiTEs, showed outstanding response rates in heavily pretreated patients $(123,134)$. However, relapses occur limiting the efficacy of this promising treatment approach. Lower expression of the targeted antigen on cell surface have been suggested as a possible mechanism of resistance to targeted mAb therapy (148, 149). Regarding CAR-T therapies, the composition and phenotypic of the administered $\mathrm{T}$ cells product, in term of the T-cells subsets, play a critical role for the clinical success of this strategy. Less differentiated (memory-like) CAR$\mathrm{T}$ cells have been linked to better expansion, long-term in vivo persistence, and sustained anti-tumor control (124). Local immune suppression and T-cell exhaustion are important mediating factors of clinical outcomes and responses to immunebased approaches (59). Therefore, further characterization of the defects present in the immune system of MM patients is essential to develop novel therapies and to repurpose the existing ones.

Further research is now required to define the most active and safe combination and the most appropriate time point of drugs administration throughout the course of the disease. Although most of the immune-based studies were completed in RRMM patients, it is expected that patients benefit the most when it is used earlier in their disease course. The optimal sequence of the different type of immune therapies is also unspecified and in need of further studies.

Lastly, the detection of prognostic factors or biomarkers able to predict clinical responses and/or toxicity in patients will enable more active tailored treatments and better survival for MM patients. As such interrogation at single cell level of the BM immune repertoire of patients treated with immunotherapies can identify cellular mediators of sensitivity or resistance to those therapies and define potential means to reinstate sensitivity. Along with the improvement of existing therapeutic strategies and the development of new approaches, a better understanding of the role of immune system in MM pathogenesis is essential.

\section{AUTHOR CONTRIBUTIONS}

$\mathrm{NL}, \mathrm{RM}, \mathrm{FH}$, and PN analyzed the data and wrote the manuscript.

4. Rutella S, Locatelli F. Targeting multiple-myeloma-induced immune dysfunction to improve immunotherapy outcomes. Clin Dev Immunol. (2012) 2012:196063. doi: 10.1155/2012/196063

5. Raitakari M, Brown RD, Gibson J, Joshua DE. T cells in myeloma. Hematol Oncol. (2003) 21:33-42. doi: 10.1002/hon.704

6. Chu VT, Berek C. The establishment of the plasma cell survival niche in the bone marrow. Immunol Rev. (2013) 251:177-88. doi: 10.1111/imr.12011

7. Moreau P, Attal M, Facon T. Frontline therapy of multiple myeloma. Blood. (2015) 125:3076-84. doi: 10.1182/blood-2014-09-568915

8. Nooka AK, Kastritis E, Dimopoulos MA, Lonial S. Treatment options for relapsed and refractory multiple myeloma. 
Blood. (2015) 125:3085-99. doi: 10.1182/blood-2014-11568923

9. Neri P, Bahlis NJ, Lonial S. New strategies in multiple myeloma: immunotherapy as a novel approach to treat patients with multiple myeloma. Clin Cancer Res. (2016) 22:5959-65. doi: 10.1158/1078-0432.CCR-16-0184

10. Kohler M, Greil C, Hudecek M, Lonial S, Raje N, Wasch R, et al. Current developments in immunotherapy in the treatment of multiple myeloma. Cancer. (2018) 124:2075-85. doi: 10.1002/cncr.31243

11. Bergsagel PL, Kuehl WM. Molecular pathogenesis and a consequent classification of multiple myeloma. J Clin Oncol. (2005) 23:6333-8. doi: 10.1200/JCO.2005.05.021

12. Manier S, Sacco A, Leleu X, Ghobrial IM, Roccaro AM. Bone marrow microenvironment in multiple myeloma progression. J Biomed Biotechnol. (2012) 2012:157496. doi: 10.1155/2012/157496

13. Meads MB, Hazlehurst LA, Dalton WS. The bone marrow microenvironment as a tumor sanctuary and contributor to drug resistance. Clin Cancer Res. (2008) 14:2519-26. doi: 10.1158/1078-0432.CCR-07-2223

14. Kay NE, Leong TL, Bone N, Vesole DH, Greipp PR, Van Ness B, et al. Blood levels of immune cells predict survival in myeloma patients: results of an Eastern Cooperative Oncology Group phase 3 trial for newly diagnosed multiple myeloma patients. Blood. (2001) 98:23-8. doi: 10.1182/blood.V98.1.23

15. Favaloro J, Brown R, Aklilu E, Yang S, Suen H, Hart D, et al. Myeloma skews regulatory $\mathrm{T}$ and pro-inflammatory $\mathrm{T}$ helper 17 cell balance in favor of a suppressive state. Leuk Lymphoma. (2014) 55:1090-8. doi: 10.3109/10428194.2013.825905

16. Ogawara H, Handa H, Yamazaki T, Toda T, Yoshida K, Nishimoto N, et al. High Th1/Th2 ratio in patients with multiple myeloma. Leuk Res. (2005) 29:135-40. doi: 10.1016/j.leukres.2004.06.003

17. Prabhala RH, Pelluru D, Fulciniti M, Prabhala HK, Nanjappa P, Song W, et al. Elevated IL-17 produced by TH17 cells promotes myeloma cell growth and inhibits immune function in multiple myeloma. Blood. (2010) 115:5385-92. doi: 10.1182/blood-2009-10-246660

18. Yago T, Nanke Y, Kawamoto M, Furuya T, Kobashigawa T, Kamatani N, et al. IL-23 induces human osteoclastogenesis via IL-17 in vitro, and anti-IL23 antibody attenuates collagen-induced arthritis in rats. Arthritis Res Ther. (2007) 9:R96. doi: 10.1186/ar2297

19. Brown RD, Pope B, Murray A, Esdale W, Sze DM, Gibson J, et al. Dendritic cells from patients with myeloma are numerically normal but functionally defective as they fail to up-regulate CD80 (B7-1) expression after huCD40LT stimulation because of inhibition by transforming growth factor-betal and interleukin-10. Blood. (2001) 98:2992-8. doi: 10.1182/blood.V98.10.2992

20. Brown R, Murray A, Pope B, Sze DM, Gibson J, Ho PJ, et al. Either interleukin-12 or interferon-gamma can correct the dendritic cell defect induced by transforming growth factor beta in patients with myeloma. $\mathrm{Br}$ J Haematol. (2004) 125:743-8. doi: 10.1111/j.1365-2141.2004.04984.x

21. Chauhan D, Singh AV, Brahmandam M, Carrasco R, Bandi M, Hideshima $\mathrm{T}$, et al. Functional interaction of plasmacytoid dendritic cells with multiple myeloma cells: a therapeutic target. Cancer Cell. (2009) 16:309-23. doi: 10.1016/j.ccr.2009.08.019

22. Ray A, Das DS, Song Y, Richardson P, Munshi NC, Chauhan D, et al. Targeting PD1-PDL1 immune checkpoint in plasmacytoid dendritic cell interactions with $\mathrm{T}$ cells, natural killer cells and multiple myeloma cells. Leukemia. (2015) 29:1441-4. doi: 10.1038/leu.2015.11

23. Trotta R, Dal Col J, Yu J, Ciarlariello D, Thomas B, Zhang X, et al. TGFbeta utilizes SMAD3 to inhibit CD16-mediated IFN-gamma production and antibody-dependent cellular cytotoxicity in human NK cells. J Immunol. (2008) 181:3784-92. doi: 10.4049/jimmunol.181.6.3784

24. El-Sherbiny YM, Meade JL, Holmes TD, McGonagle D, Mackie SL, Morgan AW, et al. The requirement for DNAM-1, NKG2D, and NKp46 in the natural killer cell-mediated killing of myeloma cells. Cancer Res. (2007) 67:8444-9. doi: 10.1158/0008-5472.CAN-06-4230

25. Jinushi M, Vanneman M, Munshi NC, Tai YT, Prabhala RH, Ritz J, et al. MHC class I chain-related protein A antibodies and shedding are associated with the progression of multiple myeloma. Proc Natl Acad Sci USA. (2008) 105:1285-90. doi: 10.1073/pnas.0711293105

26. Bonanno G, Mariotti A, Procoli A, Folgiero V, Natale D, De Rosa L, et al. Indoleamine 2,3-dioxygenase 1 (IDO1) activity correlates with immune system abnormalities in multiple myeloma. J Transl Med. (2012) 10:247. doi: 10.1186/1479-5876-10-247

27. Rebmann V, Schutt P, Brandhorst D, Opalka B, Moritz T, Nowrousian MR, et al. Soluble MICA as an independent prognostic factor for the overall survival and progression-free survival of multiple myeloma patients. Clin Immunol. (2007) 123:114-20. doi: 10.1016/j.clim.2006.11.007

28. Katz JB, Muller AJ, Prendergast GC. Indoleamine 2,3-dioxygenase in T-cell tolerance and tumoral immune escape. Immunol Rev. (2008) 222:206-21. doi: 10.1111/j.1600-065X.2008.00610.x

29. Zou W. Regulatory $\mathrm{T}$ cells, tumour immunity and immunotherapy. Nat Rev Immunol. (2006) 6:295-307. doi: 10.1038/nri1806

30. Berardi S, Ria R, Reale A, De Luisi A, Catacchio I, Moschetta M, et al. Multiple myeloma macrophages: pivotal players in the tumor microenvironment. J Oncol. (2013) 2013:183602. doi: 10.1155/2013/183602

31. Ramachandran IR, Martner A, Pisklakova A, Condamine T, Chase T, Vogl T, et al. Myeloid-derived suppressor cells regulate growth of multiple myeloma by inhibiting $\mathrm{T}$ cells in bone marrow. J Immunol. (2013) 190:3815-23. doi: 10.4049/jimmunol.1203373

32. Gabrilovich DI, Nagaraj S. Myeloid-derived suppressor cells as regulators of the immune system. Nat Rev Immunol. (2009) 9:162-74. doi: $10.1038 /$ nri2506

33. Gorgun GT, Whitehill G, Anderson JL, Hideshima T, Maguire C, Laubach $\mathrm{J}$, et al. Tumor-promoting immune-suppressive myeloid-derived suppressor cells in the multiple myeloma microenvironment in humans. Blood. (2013) 121:2975-87. doi: 10.1182/blood-2012-08-448548

34. Huang B, Pan PY, Li Q, Sato AI, Levy DE, Bromberg J, et al. Gr-1+CD115+ immature myeloid suppressor cells mediate the development of tumorinduced $\mathrm{T}$ regulatory cells and T-cell anergy in tumor-bearing host. Cancer Res. (2006) 66:1123-31. doi: 10.1158/0008-5472.CAN-05-1299

35. Wherry EJ. T cell exhaustion. Nat Immunol. (2011) 12:492-9. doi: 10.1038/ni.2035

36. Suen H, Brown R, Yang S, Weatherburn C, Ho PJ, Woodland N, et al. Multiple myeloma causes clonal T-cell immunosenescence: identification of potential novel targets for promoting tumour immunity and implications for checkpoint blockade. Leukemia. (2016) 30:1716-24. doi: 10.1038/leu.2016.84

37. Zelle-Rieser C, Thangavadivel S, Biedermann R, Brunner A, Stoitzner P, Willenbacher $\mathrm{E}$, et al. $\mathrm{T}$ cells in multiple myeloma display features of exhaustion and senescence at the tumor site. J Hematol Oncol. (2016) 9:116. doi: 10.1186/s13045-016-0345-3

38. Minnie SA, Kuns RD, Gartlan KH, Zhang P, Wilkinson AN, Samson L, et al. Myeloma escape after stem cell transplantation is a consequence of T-cell exhaustion and is prevented by TIGIT blockade. Blood. (2018) 132:1675-88. doi: 10.1182/blood-2018-01-825240

39. Dimopoulos M, Spencer A, Attal M, Prince HM, Harousseau JL, Dmoszynska $\mathrm{A}$, et al. Lenalidomide plus dexamethasone for relapsed or refractory multiple myeloma. N Engl J Med. (2007) 357:2123-32. doi: 10.1056/NEJMoa070594

40. Weber DM, Chen C, Niesvizky R, Wang M, Belch A, Stadtmauer EA, et al. Lenalidomide plus dexamethasone for relapsed multiple myeloma in North America. N Engl J Med. (2007) 357:2133-42. doi: 10.1056/NEJMoa070596

41. Miguel JS, Weisel K, Moreau P, Lacy M, Song K, Delforge M, et al. Pomalidomide plus low-dose dexamethasone vs. high-dose dexamethasone alone for patients with relapsed and refractory multiple myeloma (MM-003): a randomised, open-label, phase 3 trial. Lancet Oncol. (2013) 14:1055-66. doi: 10.1016/S1470-2045(13)70380-2

42. Quach H, Ritchie D, Stewart AK, Neeson P, Harrison S, Smyth MJ, et al. Mechanism of action of immunomodulatory drugs (IMiDS) in multiple myeloma. Leukemia. (2010) 24:22-32. doi: 10.1038/leu.2009.236

43. Lu G, Middleton RE, Sun H, Naniong M, Ott CJ, Mitsiades CS, et al. The myeloma drug lenalidomide promotes the cereblon-dependent destruction of Ikaros proteins. Science. (2014) 343:305-9. doi: 10.1126/science.12 44917

44. Kronke J, Udeshi ND, Narla A, Grauman P, Hurst SN, McConkey M, et al. Lenalidomide causes selective degradation of IKZF1 and IKZF3 in multiple myeloma cells. Science. (2014) 343:301-5. doi: 10.1126/science.1244851

45. Petzold G, Fischer ES, Thoma NH. Structural basis of lenalidomide-induced CK1alpha degradation by the CRL4(CRBN) ubiquitin ligase. Nature. (2016) 532:127-30. doi: 10.1038/nature16979 
46. LeBlanc R, Hideshima T, Catley LP, Shringarpure R, Burger R, Mitsiades $\mathrm{N}$, et al. Immunomodulatory drug costimulates $\mathrm{T}$ cells via the B7-CD28 pathway. Blood. (2004) 103:1787-90. doi: 10.1182/blood-2003-02-0361

47. Davies FE, Raje N, Hideshima T, Lentzsch S, Young G, Tai YT, et al. Thalidomide and immunomodulatory derivatives augment natural killer cell cytotoxicity in multiple myeloma. Blood. (2001) 98:210-6. doi: 10.1182/blood.V98.1.210

48. Galustian C, Meyer B, Labarthe MC, Dredge K, Klaschka D, Henry $\mathrm{J}$, et al. The anti-cancer agents lenalidomide and pomalidomide inhibit the proliferation and function of $\mathrm{T}$ regulatory cells. Cancer Immunol Immunother. (2009) 58:1033-45. doi: 10.1007/s00262-008-0620-4

49. Lagrue K, Carisey A, Morgan DJ, Chopra R, Davis DM. Lenalidomide augments actin remodeling and lowers NK-cell activation thresholds. Blood. (2015) 126:50-60. doi: 10.1182/blood-2015-01-625004

50. Sehgal K, Das R, Zhang L, Verma R, Deng Y, Kocoglu M, et al. Clinical and pharmacodynamic analysis of pomalidomide dosing strategies in myeloma: impact of immune activation and cereblon targets. Blood. (2015) 125:404251. doi: 10.1182/blood-2014-11-611426

51. Henry JY, Labarthe MC, Meyer B, Dasgupta P, Dalgleish AG, Galustian C. Enhanced cross-priming of naive CD8 $+\mathrm{T}$ cells by dendritic cells treated by the IMiDs(R) immunomodulatory compounds lenalidomide and pomalidomide. Immunology. (2013) 139:377-85. doi: 10.1111/imm.12087

52. Dimopoulos MA, Dytfeld D, Grosicki S, Moreau P, Takezako N, Hori M, et al. Elotuzumab plus pomalidomide and dexamethasone for multiple myeloma. N Engl J Med. (2018) 379:1811-22. doi: 10.1056/NEJMoa1805762

53. Lonial S, Dimopoulos M, Palumbo A, White D, Grosicki S, Spicka I, et al. Elotuzumab therapy for relapsed or refractory multiple myeloma. $N$ Engl J Med. (2015) 373:621-31. doi: 10.1056/NEJMoa1505654

54. Dimopoulos MA, Oriol A, Nahi H, San-Miguel J, Bahlis NJ, Usmani SZ, et al. Daratumumab, Lenalidomide, and Dexamethasone for Multiple Myeloma. N Engl J Med. (2016) 375:1319-31. doi: 10.1056/NEJMoa1607751

55. Dimopoulos MA, San-Miguel J, Belch A, White D, Benboubker L, Cook G, et al. Daratumumab plus lenalidomide and dexamethasone vs. lenalidomide and dexamethasone in relapsed or refractory multiple myeloma: updated analysis of POLLUX. Haematologica. (2018) doi: 10.3324/haematol.2018.194282

56. Attal M, Richardson PG, Rajkumar SV, San-Miguel J, Beksac M, Spicka I, et al. Isatuximab plus pomalidomide and low-dose dexamethasone vs. pomalidomide and low-dose dexamethasone in patients with relapsed and refractory multiple myeloma (ICARIA-MM): a randomised, multicentre, open-label, phase 3 study. Lancet. (2019) 394:2096-107. doi: 10.1016/S0140-6736(19)32556-5

57. de Weers M, Tai YT, van der Veer MS, Bakker JM, Vink T, Jacobs DC, et al. Daratumumab, a novel therapeutic human CD38 monoclonal antibody, induces killing of multiple myeloma and other hematological tumors. $J$ Immunol. (2011) 186:1840-8. doi: 10.4049/jimmunol.1003032

58. Overdijk MB, Verploegen S, Bogels $M$, van Egmond $M$, Lammerts van Bueren JJ, Mutis T, et al. Antibody-mediated phagocytosis contributes to the anti-tumor activity of the therapeutic antibody daratumumab in lymphoma and multiple myeloma. MAbs. (2015) 7:311-21. doi: 10.1080/19420862.2015.1007813

59. Neri P, Alberge J-B, Maity R, McCulloch S, Tay J, Jimenez-Zepeda V, et al. Expansion of effector memory CD27+ T cells and tolerogenic type 2 classical dendritic cells regulate myeloma patients' sensitivity to daratumumab \& IMiDs. Clin Lymph Myeloma Leuk. (2019) 19:e29-e30. doi: 10.1016/j.clml.2019.09.044

60. Sharpe AH, Abbas AK. T-cell costimulation-biology, therapeutic potential, and challenges. N Engl J Med. (2006) 355:973-5. doi: 10.1056/NEJMp068087

61. Fox BA, Schendel DJ, Butterfield LH, Aamdal S, Allison JP, Ascierto PA, et al. Defining the critical hurdles in cancer immunotherapy. J Transl Med. (2011) 9:214. doi: 10.1186/1479-5876-9-214

62. Quezada SA, Peggs KS. Exploiting CTLA-4, PD-1 and PD-L1 to reactivate the host immune response against cancer. Br J Cancer. (2013) 108:1560-5. doi: 10.1038/bjc.2013.117

63. Pardoll DM. The blockade of immune checkpoints in cancer immunotherapy. Nat Rev Cancer. (2012) 12:252-64. doi: 10.1038/ nrc3239

64. Sharma P, Allison JP. Immune checkpoint targeting in cancer therapy: toward combination strategies with curative potential. Cell. (2015) 161:20514. doi: 10.1016/j.cell.2015.03.030
65. Yousef S, Marvin J, Steinbach M, Langemo A, Kovacsovics T, Binder M, et al. Immunomodulatory molecule PD-L1 is expressed on malignant plasma cells and myeloma-propagating pre-plasma cells in the bone marrow of multiple myeloma patients. Blood Cancer J. (2015) 5:e285. doi: 10.1038/bcj.2015.7

66. Lesokhin AM, Ansell SM, Armand P, Scott EC, Halwani A, Gutierrez M, et al. Nivolumab in patients with relapsed or refractory hematologic malignancy: preliminary results of a phase Lb study. J Clin Oncol. (2016) 34:2698-704. doi: 10.1200/JCO.2015.65.9789

67. Suen H, Brown R, Yang S, Ho PJ, Gibson J, Joshua D. The failure of immune checkpoint blockade in multiple myeloma with PD-1 inhibitors in a phase 1 study. Leukemia. (2015) 29:1621-2. doi: 10.1038/leu.2015.104

68. Badros A, Hyjek E, Ma N, Lesokhin A, Dogan A, Rapoport AP, et al. Pembrolizumab, pomalidomide, and low-dose dexamethasone for relapsed/refractory multiple myeloma. Blood. (2017) 130:1189-97. doi: 10.1182/blood-2017-03-775122

69. San Miguel J, Mateos M-V, Shah JJ, Ocio EM, Rodriguez-Otero P, Reece $\mathrm{D}$, et al. Pembrolizumab in combination with lenalidomide and lowdose dexamethasone for relapsed/refractory multiple myeloma (RRMM): keynote-023. Blood. (2015) 126:505. doi: 10.1182/blood.V126.23.505.505

70. Jing W, Gershan JA, Weber J, Tlomak D, McOlash L, Sabatos-Peyton C, et al. Combined immune checkpoint protein blockade and low dose whole body irradiation as immunotherapy for myeloma. J Immunother Cancer. (2015) 3:2. doi: 10.1186/s40425-014-0043-z

71. Yadav M, Green C, Ma C, Robert A, Glibicky A, Nakamura R, et al. Tigit, CD226 and PD-L1/PD-1 are highly expressed by marrow-infiltrating $\mathrm{T}$ cells in patients with multiple myeloma. Blood. (2016) 128:2102. doi: 10.1182/blood.V128.22.2102.2102

72. Neri P, Maity R, Tagoug I, Duggan P, McCulloch S, Jimenez-Zepeda $\mathrm{V}$, et al. Single cell resolution profiling defines the innate and adaptive immune repertoires modulated by daratumumab and IMiDs treatment in multiple myeloma (MM). Blood. (2017) 130(Suppl 1):123. doi: 10.1182/blood.V130.Suppl_1.123.123

73. Lozano E, Diaz T, Mena MP, Sune G, Calvo X, Calderon M, et al. Loss of the immune checkpoint CD85j/LILRB1 on malignant plasma cells contributes to immune escape in multiple myeloma. J Immunol. (2018) 200:2581-91. doi: 10.4049/jimmunol.1701622

74. Ghoneim HE, Fan Y, Moustaki A, Abdelsamed HA, Dash P, Dogra P, et al. De novo epigenetic programs inhibit PD-1 blockade-mediated T cell rejuvenation. Cell. (2017) 170:142-57 e19. doi: 10.1016/j.cell.2017.06.007

75. Heninger E, Krueger TE, Lang JM. Augmenting antitumor immune responses with epigenetic modifying agents. Front Immunol. (2015) 6:29. doi: 10.3389/fimmu.2015.00029

76. Stone ML, Chiappinelli KB, Li H, Murphy LM, Travers ME, Topper MJ, et al. Epigenetic therapy activates type I interferon signaling in murine ovarian cancer to reduce immunosuppression and tumor burden. Proc Natl Acad Sci USA. (2017) 114:E10981-E90. doi: 10.1073/pnas.1712514114

77. Toor AA, Payne KK, Chung HM, Sabo RT, Hazlett AF, Kmieciak M, et al. Epigenetic induction of adaptive immune response in multiple myeloma: sequential azacitidine and lenalidomide generate cancer testis antigen-specific cellular immunity. Br J Haematol. (2012) 158:700-11. doi: 10.1111/j.1365-2141.2012.09225.x

78. Moreno-Bost A, Szmania S, Stone K, Garg T, Hoerring A, Szymonifka J, et al. Epigenetic modulation of MAGE-A3 antigen expression in multiple myeloma following treatment with the demethylation agent 5-azacitidine and the histone deacetlyase inhibitor MGCD0103. Cytotherapy. (2011) 13:618-28. doi: 10.3109/14653249.2010.529893

79. De Beck L, Melhaoui S, De Veirman K, Menu E, De Bruyne E, Vanderkerken $\mathrm{K}$, et al. Epigenetic treatment of multiple myeloma mediates tumor intrinsic and extrinsic immunomodulatory effects. Oncoimmunology. (2018) 7:e1484981. doi: 10.1080/2162402X.2018.1484981

80. Hsi ED, Steinle R, Balasa B, Szmania S, Draksharapu A, Shum BP, et al. CS1, a potential new therapeutic antibody target for the treatment of multiple myeloma. Clin Cancer Res. (2008) 14:2775-84.

81. Collins SM, Bakan CE, Swartzel GD, Hofmeister CC, Efebera YA, Kwon $\mathrm{H}$, et al. Elotuzumab directly enhances NK cell cytotoxicity against myeloma via CS1 ligation: evidence for augmented NK cell function complementing ADCC. Cancer Immunol Immunother. (2013) 62:1841-9. doi: 10.1007/s00262-013-1493-8

82. Dimopoulos MA, Lonial S, White D, Moreau P, Palumbo A, San Miguel J, et al. Eloquent-2 update: a phase 3, randomized, open-label study of 
elotuzumab in combination with lenalidomide/dexamethasone in patients with relapsed/refractory multiple myeloma - 3-year safety and efficacy follow-up. Blood. (2015) 126:28. doi: 10.1182/blood.V126.23.28.28

83. Dimopoulos MA, Lonial S, Betts KA, Chen C, Zichlin ML, Brun A, et al. Elotuzumab plus lenalidomide and dexamethasone in relapsed/refractory multiple myeloma: Extended 4-year follow-up and analysis of relative progression-free survival from the randomized ELOQUENT-2 trial. Cancer. (2018) 124:4032-43. doi: $10.1002 / \mathrm{cncr} .31680$

84. Gavriatopoulou M, Terpos E, Dimopoulos MA. The extended 4-year follow-up results of the ELOQUENT-2 trial. Oncotarget. (2019) 10:82-3. doi: 10.18632/oncotarget.26527

85. Jakubowiak AJ, Benson DM, Bensinger W, Siegel DSD, Zimmerman TM, Mohrbacher A, et al. Phase I trial of anti-CS1 monoclonal antibody elotuzumab in combination with bortezomib in the treatment of relapsed/refractory multiple myeloma. J Clin Oncol. (2012) 30:1960-5. doi: 10.1200/JCO.2011.37.7069

86. Rosebeck S, Alonge MM, Kandarpa M, Mayampurath A, Volchenboum SL, Jasielec J, et al. Synergistic myeloma cell death via novel intracellular activation of caspase-10-dependent apoptosis by carfilzomib and selinexor. Mol Cancer Ther. (2016) 15:60-71. doi: 10.1158/1535-7163.MCT-15-0488

87. Malavasi F, Funaro A, Roggero S, Horenstein A, Calosso L, Mehta K. Human CD38: a glycoprotein in search of a function. Immunology Today. (1994) 15:95-7. doi: 10.1016/0167-5699(94)90148-1

88. Deaglio S, Vaisitti T, Billington R, Bergui L, Omede P, Genazzani AA, et al. CD38/CD19: a lipid raft-dependent signaling complex in human B cells. Blood. (2007) 109:5390-8. doi: 10.1182/blood-2006-12-061812

89. Malavasi F, Deaglio S, Funaro A, Ferrero E, Horenstein AL, Ortolan $\mathrm{E}$, et al. Evolution and function of the ADP ribosyl cyclase/CD38 gene family in physiology and pathology. (2008). p. 841-86. doi: 10.1152/physrev.00035.2007

90. Overdijk MB, Jansen JHM, Nederend M, Bueren JJLv, Groen RWJ, Parren PWHI, et al. The therapeutic CD38 monoclonal antibody daratumumab induces programmed cell death via Fc $\gamma$ receptor-mediated cross-linking. $J$ Immunol. (2016) 197:807-13. doi: 10.4049/jimmunol.1501351

91. Krejcik J, Casneuf T, Nijhof IS, Verbist B, Bald J, Plesner T, et al. Daratumumab depletes CD38+ immune regulatory cells, promotes T-cell expansion, and skews T-cell repertoire in multiple myeloma. Blood. (2016) 128:384-94. doi: 10.1182/blood-2015-12-687749

92. Lokhorst HM, Plesner T, Laubach JP, Nahi H, Gimsing P, Hansson M, et al. Targeting CD38 with daratumumab monotherapy in multiple myeloma. $\mathrm{N}$ Engl J Med. (2015) 373:1207-19. doi: 10.1056/NEJMoa1506348

93. Lonial S, Weiss BM, Usmani SZ, Singhal S, Chari A, Bahlis NJ, et al. Daratumumab monotherapy in patients with treatment-refractory multiple myeloma (SIRIUS): an open-label, randomised, phase 2 trial. Lancet. (2016) 387:1551-60. doi: 10.1016/S0140-6736(15)01120-4

94. Usmani SZ, Weiss BM, Plesner T, Bahlis NJ, Belch A, Lonial S, et al. Clinical efficacy of daratumumab monotherapy in patients with heavily pretreated relapsed or refractory multiple myeloma. Blood. (2016) 128:37-44. doi: 10.1182/blood-2016-03-705210

95. Bahlis N, editor. First Analysis from a Phase 1/2 Study of Venetoclax in Combination with Daratumumab and Dexamethasone, +/- Bortezomib, in Patients with Relapsed/Refractory Multiple Myeloma. 61st Annual Meeting and Exposition (December 7-10, 2019) (2019).

96. Chari A, Suvannasankha A, Fay JW, Arnulf B, Kaufman JL, Ifthikharuddin $\mathrm{JJ}$, et al. Daratumumab plus pomalidomide and dexamethasone in relapsed and/or refractory multiple myeloma. Blood. (2017) 130:974-81. doi: 10.1182/blood-2017-05-785246

97. Palumbo A, Chanan-Khan A, Weisel K, Nooka AK, Masszi T, Beksac M, et al. Daratumumab, bortezomib, and dexamethasone for multiple myeloma. $N$ Engl J Med. (2016) 375:754-66. doi: 10.1056/NEJMoa1606038

98. Spencer A, Lentzsch S, Weisel K, Avet-Loiseau H, Mark TM, Spicka I, et al. Daratumumab plus bortezomib and dexamethasone vs. bortezomib and dexamethasone in relapsed or refractory multiple myeloma: updated analysis of CASTOR. Haematologica. (2018) 103:2079-87.

99. Mateos M-V, editor. Daratumumab Plus Bortezomib, Melphalan, and Prednisone vs. Bortezomib, Melphalan, and Prednisone in Patients with Transplant-Ineligible Newly Diagnosed Multiple Myeloma: Overall Survival in Alcyone. 61st Annual Meeting and Exposition (December 7-10, 2019) (2019).
100. Chari A, Martinez-Lopez J, Mateos M-V, Bladé J, Benboubker L, Oriol A, et al. Daratumumab plus carfilzomib and dexamethasone in patients with relapsed or refractory multiple myeloma. Blood. (2019) 134:421-31. doi: 10.1182/blood.2019000722

101. Usmani S, editor. Carfilzomib, Dexamethasone, and Daratumumab vs. Carfilzomib and Dexamethasone for the Treatment of Patients with Relapsed or Refractory Multiple Myeloma (RRMM): Primary Analysis Results from the Randomized, Open-Label, Phase 3 Study Candor (NCT03158688). 61st Annual Meeting and Exposition (December 7-10, 2019); (2019).

102. Mateos M-V, Dimopoulos MA, Cavo M, Suzuki K, Jakubowiak A, Knop S, et al. Daratumumab plus bortezomib, melphalan, and prednisone for untreated myeloma. N Engl J Med. (2018) 378:518-28. doi: 10.1056/NEJMoa1714678

103. Facon T, Kumar S, Plesner T, Orlowski RZ, Moreau P, Bahlis N, et al. Daratumumab plus lenalidomide and dexamethasone for untreated myeloma. N Engl J Med. (2019) 380:2104-15. doi: 10.1056/NEJMoa1817249

104. Moreau P, Attal M, Hulin C, Arnulf B, Belhadj K, Benboubker $\mathrm{L}$, et al. Bortezomib, thalidomide, and dexamethasone with or without daratumumab before and after autologous stem-cell transplantation for newly diagnosed multiple myeloma (CASSIOPEIA): a randomised, open-label, phase 3 study. Lancet. (2019) 394:29-38. doi: 10.1016/S0140-6736(19)31240-1

105. Voorhees P, editor. Depth of Response to Daratumumab (DARA), Lenalidomide, Bortezomib, and Dexamethasone (RVd) Improves over Time in Patients (pts) with Transplant-Eligible Newly Diagnosed Multiple Myeloma (NDMM): Griffin Study Update. 61st Annual Meeting and Exposition (December 7-10, 2019); (2019).

106. Moreno L, Pérez C, Zabaleta A, Manrique I, Alignani D, Ajona D, et al. The mechanism of action of the anti-CD38 monoclonal antibody isatuximab in multiple myelmoa. Clin Cancer Res. (2019) 25:3176-87. doi: 10.1158/1078-0432.CCR-18-1597

107. Martin T, Strickland S, Glenn M, Charpentier E, Guillemin H, Hsu $\mathrm{K}$, et al. Phase I trial of isatuximab monotherapy in the treatment of refractory multiple myeloma. Blood Cancer J. (2019) 9:1-10. doi: 10.1038/s41408-019-0198-4

108. Martin T, Baz R, Benson DM, Lendvai N, Wolf J, Munster P, et al. A phase $1 \mathrm{~b}$ study of isatuximab plus lenalidomide and dexamethasone for relapsed/refractory multiple myeloma. Blood. (2017) 129:3294-303. doi: 10.1182/blood-2016-09-740787

109. Mikhael J, Richardson P, Usmani SZ, Raje N, Bensinger W, Karanes C, et al. A phase $1 \mathrm{~b}$ study of isatuximab plus pomalidomide/dexamethasone in relapsed/refractory multiple myeloma. Blood. (2019) 134:123-33. doi: 10.1182/blood-2019-02-895193

110. Moreau P, Dimopoulos MA, Yong K, Mikhael J, Risse M-L, Asset G, et al. Isatuximab plus carfilzomib/dexamethasone vs. carfilzomib/dexamethasone in patients with relapsed/refractory multiple myeloma: IKEMA Phase III study design. Fut Oncol. (2019) 16:4347-58. doi: 10.2217/fon-2019-0431

111. Raab MS, Chatterjee M, Goldschmidt H, Agis H, Blau I, Einsele H, et al. MOR202 with low-dose dexamethasone (dex) or pomalidomide/dex or lenalidomide/dex in relapsed or refractory multiple myeloma (RRMM): primary analysis of a phase i/iia, multicenter, dose-escalation study. Blood. (2018) 132(Supplement 1):153. doi: 10.1182/blood-2018-153

112. Krishnan A, editor. Preliminary Results from a Phase $1 b$ Study of TAK079, an Investigational Anti-CD38 Monoclonal Antibody ( $m A b)$ in Patients with Relapsed/ Refractory Multiple Myeloma (RRMM). 61st Annual Meeting Exposition (December 7-10, 2019) (2019). doi: 10.1182/blood-2019-128007

113. Mikkilineni L, Kochenderfer JN. Chimeric antigen receptor Tcell therapies for multiple myeloma. Blood. (2017) 130:2594-602. doi: 10.1182/blood-2017-06-793869

114. Dotti G, Gottschalk S, Savoldo B, Brenner MK. Design and development of therapies using chimeric antigen receptor-expressing $\mathrm{T}$ cells. Immunol Rev. (2014) 257:107-26. doi: 10.1111/imr.12131

115. Wu C, Zhang L, Brockman QR, Zhan F, Chen L. Chimeric antigen receptor T cell therapies for multiple myeloma. J Hematol Oncol. (2019) 12:120. doi: 10.1186/s13045-019-0823-5

116. Timmers M, Roex G, Wang Y, Campillo-Davo D, Van Tendeloo VFI, Chu $\mathrm{Y}$, et al. Chimeric antigen receptor-modified $\mathrm{T}$ cell therapy in multiple myeloma: beyond $\mathrm{B}$ cell maturation antigen. 
Front Immunol. (2019) 10:1613. doi: 10.3389/fimmu.2019. 01613

117. Rickert RC, Jellusova J, Miletic AV. Signaling by the tumor necrosis factor receptor superfamily in B-cell biology and disease. Immunol Rev. (2011) 244:115-33. doi: 10.1111/j.1600-065X.2011.01067.x

118. Laurent SA, Hoffmann FS, Kuhn P-H, Cheng Q, Chu Y, Schmidt-Supprian $\mathrm{M}$, et al. $\gamma$-Secretase directly sheds the survival receptor BCMA from plasma cells. Nat Commun. (2015). 6:7333. doi: 10.1038/ncomms8333

119. Sanchez E, Li M, Kitto A, Li J, Wang CS, Kirk DT, et al. Serum Bcell maturation antigen is elevated in multiple myeloma and correlates with disease status and survival. Br J Haematol. (2012) 158:727-38. doi: 10.1111/j.1365-2141.2012.09241.x

120. Tai Y-T, Acharya C, An G, Moschetta M, Zhong MY, Feng X, et al. APRIL and BCMA promote human multiple myeloma growth and immunosuppression in the bone marrow microenvironment. Blood. (2016) 127:3225-36. doi: 10.1182/blood-2016-01-691162

121. Brudno JN, Maric I, Hartman SD, Rose JJ, Wang M, Lam N, et al. T cells genetically modified to express an anti-B-cell maturation antigen chimeric antigen receptor cause remissions of poor-prognosis relapsed multiple myeloma. J Clin Oncol. (2018) 36:2267-80. doi: 10.1200/JCO.2018.77.8084

122. Cohen AD, Garfall AL, Stadtmauer EA, Melenhorst JJ, Lacey SF, Lancaster $\mathrm{E}$, et al. B cell maturation antigen-specific CAR T cells are clinically active in multiple myeloma. J Clin Invest. (2019) 129:2210-21. doi: 10.1172/JCI126397

123. Raje N, Berdeja J, Lin Y, Siegel D, Jagannath S, Madduri D, et al. Anti-BCMA CAR T-Cell Therapy bb2121 in Relapsed or Refractory Multiple Myeloma. $N$ Engl J Med. (2019) 380:1726-37. doi: 10.1056/NEJMoa1817226

124. Shah N, Alsina M, Siegel DS, Jagannath S, Madduri D, Kaufman JL, et al. Initial results from a phase 1 clinical study of bb21217, a nextgeneration anti Bcma CAR T therapy. Blood. (2018) 132(Supplement 1):488. doi: 10.1182/blood-2018-99-116953

125. Zhao W-H, Liu J, Wang B-Y, Chen Y-X, Cao X-M, Yang Y, et al. A phase 1, open-label study of LCAR-B38M, a chimeric antigen receptor T cell therapy directed against B cell maturation antigen, in patients with relapsed or refractory multiple myeloma. J Hematol Oncol. (2018) 11:141. doi: 10.1186/s13045-018-0681-6

126. Xu J, Chen L-J, Yang S-S, Sun Y, Wu W, Liu Y-F, et al. Exploratory trial of a biepitopic CAR T-targeting $\mathrm{B}$ cell maturation antigen in relapsed/refractory multiple myeloma. PNAS. (2019) 116:9543-51. doi: $10.1073 /$ pnas. 1819745116

127. Tolcher AW. Antibody drug conjugates: lessons from 20 years of clinical experience. Ann Oncol. (2016) 27:2168-72. doi: 10.1093/annonc/mdw424

128. Trudel S, Lendvai N, Popat R, Voorhees PM, Reeves B, Libby EN, et al. Targeting B-cell maturation antigen with GSK2857916 antibody-drug conjugate in relapsed or refractory multiple myeloma (BMA117159): a dose escalation and expansion phase 1 trial. Lancet Oncol. (2018) 19:1641-53. doi: 10.1016/S1470-2045(18)30576-X

129. Klinger M, Benjamin J, Kischel R, Stienen S, Zugmaier G. Harnessing T cells to fight cancer with BiTE $\AA$ antibody constructs - past developments and future directions. Immunol Rev. (2016) 270:193-208. doi: 10.1111/imr.12393

130. Baeuerle PA, Reinhardt C. Bispecific T-cell engaging antibodies for cancer therapy. Cancer Res. (2009) 69:4941-4. doi: 10.1158/0008-5472.CAN-09-0547

131. Frankel SR, Baeuerle PA. Targeting $\mathrm{T}$ cells to tumor cells using bispecific antibodies. Curr Opin Chem Biol. (2013) 17:385-92. doi: 10.1016/j.cbpa.2013.03.029

132. Topp MS, Gökbuget N, Stein AS, Zugmaier G, O’Brien S, Bargou RC, et al. Safety and activity of blinatumomab for adult patients with relapsed or refractory B-precursor acute lymphoblastic leukaemia: a multicentre, single-arm, phase 2 study. Lancet Oncol. (2015) 16:57-66. doi: 10.1016/S1470-2045(14)71170-2

133. Hipp S, Tai YT, Blanset D, Deegen P, Wahl J, Thomas O, et al. A novel BCMA/CD3 bispecific T-cell engager for the treatment of multiple myeloma induces selective lysis in vitro and in vivo. Leukemia. (2017) 31:1743-51. doi: 10.1038/leu.2016.388

134. Topp MS, Duell J, Zugmaier G, Attal M, Moreau P, Langer C, et al. Anti-B-Cell Maturation Antigen BiTE Molecule AMG 420 Induces Responses in Multiple Myeloma. J Clin Oncol. 2020:JCO1902657. doi: 10.1200/JCO.19.02657
135. Cho S-F, editor. AMG 701 Potently Induces Anti-Multiple Myeloma (MM) Functions of T Cells and IMiDs Further Enhance Its Efficacy to Prevent MM Relapse In Vivo. 61st Annual Meeting and Exposition (December 7-10, 2019) (2019).

136. Law C-L, Aaron W, Austin R, Barath M, Callihan E, Evans T, et al. Preclinical and nonclinical characterization of HPN217: a tri-specific T cell activating construct (TriTAC) targeting B cell maturation antigen (BCMA) for the treatment of multiple myeloma. Blood. (2018) 132(Supplement 1):3225. doi: 10.1182/blood-2018-99-113921

137. $\mathrm{Hu} \mathrm{Z}$, Ott PA, Wu CJ. Towards personalized, tumour-specific, therapeutic vaccines for cancer. Nat Rev Immunol. (2018) 18:168-82. doi: $10.1038 /$ nri.2017.131

138. Wong KK, Li WA, Mooney DJ, Dranoff G. Advances in Therapeutic Cancer Vaccines. Adv Immunol. (2016) 130:191-249. doi: 10.1016/bs.ai.2015.12.001

139. Gilboa E. The promise of cancer vaccines. Nat Rev Cancer. (2004) 4:401-11. doi: $10.1038 / \mathrm{nrc} 1359$

140. Mougel A, Terme M, Tanchot C. Therapeutic cancer vaccine and combinations with antiangiogenic therapies and immune checkpoint blockade. Front Immunol. (2019) 10:467. doi: 10.3389/fimmu.2019.00467

141. Rosenblatt J, Avigan D. Cellular immunotherapy for multiple myeloma. Cancer J. (2019) 25:38-44. doi: 10.1097/PPO.0000000000000356

142. Rapoport AP, Aqui NA, Stadtmauer EA, Vogl DT, Fang H-B, Cai L, et al. Combination immunotherapy using adoptive T-cell transfer and tumor antigen vaccination on the basis of hTERT and survivin after ASCT for myeloma. Blood. (2011) 117:788-97. doi: 10.1182/blood-2010-08-299396

143. Rapoport AP, Aqui NA, Stadtmauer EA, Vogl DT, Xu YY, Kalos M, et al. Combination immunotherapy after ASCT for multiple myeloma using MAGE-A3/Poly-ICLC immunizations followed by adoptive transfer of vaccine-primed and costimulated autologous T cells. Clin Cancer Res. (2014) 20:1355-65. doi: 10.1158/1078-0432.CCR-13-2817

144. Cohen AD, Lendvai N, Nataraj S, Imai N, Jungbluth AA, Tsakos I, et al. Autologous lymphocyte infusion supports tumor antigen vaccine-induced immunity in autologous stem cell transplant for multiple myeloma. Cancer Immunol Res. (2019) 7:658-69. doi: 10.1158/2326-6066.CIR-18-0198

145. Nooka AK, Wang ML, Yee AJ, Kaufman JL, Bae J, Peterkin D, et al. Assessment of safety and immunogenicity of PVX-410 vaccine with or without lenalidomide in patients with smoldering multiple myeloma: a nonrandomized clinical trial. JAMA Oncol. (2018) 4:e183267. doi: 10.1001/jamaoncol.2018.3267

146. Rosenblatt J, Avivi I, Vasir B, Uhl L, Munshi NC, Katz T, et al. Vaccination with Dendritic Cell/Tumor Fusions following Autologous Stem Cell Transplant Induces Immunologic and Clinical Responses in Multiple Myeloma Patients. Clin Cancer Res. (2013) 19:3640-8. doi: 10.1158/1078-0432.CCR-13-0282

147. Mateos MV, Ludwig H, Bazarbachi A, Beksac M, Blade J, Boccadoro $M$, et al. Insights on Multiple Myeloma Treatment Strategies. Hemasphere. (2019) 3:e163. doi: 10.1097/HS9.00000000000 00163

148. Nijhof IS, Casneuf T, van Velzen J, van Kessel B, Axel AE, Syed K, et al. CD38 expression and complement inhibitors affect response and resistance to daratumumab therapy in myeloma. Blood. (2016) 128:959-70. doi: 10.1182/blood-2016-03-703439

149. Danhof S, Strifler S, Hose D, Kortum M, Bittrich M, Hefner J, et al. Clinical and biological characteristics of myeloma patients influence response to elotuzumab combination therapy. J Cancer Res Clin Oncol. (2019) 145:56171. doi: $10.1007 / \mathrm{s} 00432-018-2807-1$

Conflict of Interest: The authors declare that the research was conducted in the absence of any commercial or financial relationships that could be construed as a potential conflict of interest.

Copyright (๑) 2020 Leblay, Maity, Hasan and Neri. This is an open-access article distributed under the terms of the Creative Commons Attribution License (CC BY). The use, distribution or reproduction in other forums is permitted, provided the original author(s) and the copyright owner(s) are credited and that the original publication in this journal is cited, in accordance with accepted academic practice. No use, distribution or reproduction is permitted which does not comply with these terms. 